\title{
On Global Existence of Solutions of the Neumann Problem for Spherically Symmetric Nonlinear Viscoelasticity in a Ball
}

\author{
Jerzy A. Gawinecki ${ }^{1}$ and Wojciech M. Zajączkowski ${ }^{1,2}$ \\ ${ }^{1}$ Institute of Mathematics and Cryptology, Cybernetics Faculty, Military University of Technology, Kaliskiego 2, 00-908 Warsaw, Poland \\ ${ }^{2}$ Institute of Mathematics, Polish Academy of Sciences, Śniadeckich 8, 00-956 Warsaw, Poland \\ Correspondence should be addressed to Jerzy A. Gawinecki; jgawinecki@wat.edu.pl
}

Received 21 November 2012; Accepted 20 December 2012

Academic Editors: G. Akrivis, L. Wang, and C. Zhu

Copyright ( 2013 J. A. Gawinecki and W. M. Zajączkowski. This is an open access article distributed under the Creative Commons Attribution License, which permits unrestricted use, distribution, and reproduction in any medium, provided the original work is properly cited.

\begin{abstract}
We examine spherically symmetric solutions to the viscoelasticity system in a ball with the Neumann boundary conditions. Imposing some growth restrictions on the nonlinear part of the stress tensor, we prove the existence of global regular solutions for large data in the weighted Sobolev spaces, where the weight is a power function of the distance to the centre of the ball. First, we prove a global a priori estimate. Then existence is proved by the method of successive approximations and appropriate time extension.
\end{abstract}

\section{Introduction}

First, we recall some important facts from the nonlinear theory of viscoelasticity. Among the papers devoted to nonlinear viscoelasticity, we mention below some of them. The global solution (in time) for sufficiently small and smooth data are proved by Ponce (cf. [1]) and Kawashima and Shibata (cf. [2]) for quasilinear hyperbolic system of second-order with viscosity.

In the paper of [3], Kobayashi, Pecher, and Shibata proved global in time solution to a nonlinear wave equation with viscoelasticity under the special assumption about nonlinearity. In the paper of [4], Pawłow and Zajączkowski showed the existence and uniqueness of global regular solutions to the Cahn-Hilliard system coupled with viscoelasticity.

Finally, in [5], global existence of regular solutions to one-dimensional viscoelasticity is proved. Moreover, many facts on elasticity and viscoelasticity theory can be found in [6-10]. Some existence results in the linear and nonlinear thermoviscoelasticity are shown in $[11,12]$.

In our paper, we consider a more general nonlinear system of viscoelasticity because the stress tensor is a general nonlinear function depending on a strain. We assume that the stress tensor is a function of a strain at a given instant of time $t$, but it does not depend on strains at time $t^{\prime}<t$.
It is worth to emphasize that our constitutive relation for the stress tensor and any another constitutive relation satisfy the rules of continuum mechanics.

In order to prove the global (in time) solution for nonsmall data for nonlinear system of viscoelasticity (cf. formulae (1), (2), and (3)), we consider the spherically symmetric case and use the anisotropic Sobolev spaces with weights.

Speaking more precisely, we consider the motion of viscoelastic medium described by the following system of equations (cf. $[7,11])$ :

$$
\varrho u_{, t t}=\operatorname{di} v \sigma+\varrho f
$$

where $u=u(x, t)=\left(u_{1}(x, t), u_{2}(x, t), u_{3}(x, t)\right) \in \mathbb{R}^{3}$ is the displacement vector, $x=\left(x_{1}, x_{2}, x_{3}\right) \in \mathbb{R}^{3}$ is a given system of the Cartesian coordinates, $t \in \mathbb{R}_{+} \cup\{0\}, \varrho$ is the mass density, $\sigma=\sigma(x, t) \in \mathbb{R}^{9}$ is the stress tensor, and $f=\left(f_{1}(x, t), f_{2}(x, t), f_{3}(x, t)\right) \in \mathbb{R}^{3}$ is the external force field.

We examine system (1) in a bounded domain $\Omega \subset \mathbb{R}^{3}$ with the Neumann boundary conditions

$$
\left.\bar{n} \cdot \sigma\right|_{S}=0,
$$

where $S=\partial \Omega, \bar{n}$ is the unit outward vector normal to $S$. 
Moreover, we add the initial conditions

$$
\left.u\right|_{t=0}=u_{0},\left.\quad u_{t}\right|_{t=0}=u_{1} .
$$

We will assume that

$$
\sigma=\frac{\partial F}{\partial \varepsilon}(\varepsilon)+\mu_{0} \varepsilon_{t}
$$

where $\varepsilon=(1 / 2)\left(\nabla u+(\nabla u)^{T}\right)$ is the linearized strain tensor, $F=F(\varepsilon)$ is some function which will be specified later, and $\mu_{0}$ is a positive constant.

Our aim is to prove the global existence of solutions to problems (1)-(4) for nonsmall data.

Since we do not know how to show the existence in a general case, we restrict our considerations to the spherically symmetric case. We assume that $\Omega$ is a ball $B_{R}$ with radius $R$ centered at the origin of the introduced Cartesian coordinates. We introduce the spherical coordinates $r, \varphi, \vartheta$ by the relations

$$
\begin{gathered}
x_{1}=r \cos \varphi \sin \vartheta, \\
x_{2}=r \sin \varphi \sin \vartheta, \\
x_{3}=r \cos \vartheta .
\end{gathered}
$$

With these coordinates, we connect the orthonormal vectors

$$
\begin{gathered}
\bar{e}_{r}=(\cos \varphi \sin \vartheta, \sin \varphi \sin \vartheta, \cos \vartheta) \\
\bar{e}_{\vartheta}=(\cos \varphi \cos \vartheta, \sin \varphi \cos \vartheta,-\sin \vartheta), \\
\bar{e}_{\varphi}=(-\sin \varphi, \cos \varphi, 0) .
\end{gathered}
$$

Then, we define $u_{r}=u \cdot \bar{e}_{r}, u_{\vartheta}=u \cdot \bar{e}_{\vartheta}, u_{\varphi}=u \cdot \bar{e}_{\varphi}, \varepsilon_{r r}=$ $\bar{e}_{r} \cdot \varepsilon \cdot \bar{e}_{r}=u_{r, r}, \varepsilon_{\varphi \varphi}=u_{r} / r, \varepsilon_{\vartheta \vartheta}=u_{r} / r$. Since the spherically symmetric case is considered, we have $u_{\vartheta}=u_{\varphi}=0$.

To simplify the notation, we introduce

$$
w=u_{r}
$$

Assuming $\varrho=1$ and transforming (1) to the spherical coordinates, we obtain

$$
w_{, t t}=\frac{1}{r^{2}}\left(\sigma_{r r} r^{2}\right)_{, r}-\frac{1}{r}\left(\sigma_{\vartheta \vartheta}+\sigma_{\varphi \varphi}\right)+f_{r},
$$

where

$$
\begin{gathered}
\sigma_{r r}=\frac{\partial F}{\partial \varepsilon_{r r}}+\mu_{0} \varepsilon_{r r, t}, \quad \sigma_{\vartheta \vartheta}=\frac{\partial F}{\partial \varepsilon_{\vartheta \vartheta}}+\mu_{0} \varepsilon_{\vartheta \vartheta, t} \\
\sigma_{\varphi \varphi}=\frac{\partial F}{\partial \varepsilon_{\varphi \varphi}}+\mu_{0} \varepsilon_{\varphi \varphi, t} .
\end{gathered}
$$

Let us introduce the quantity

$$
F(\varepsilon)=\psi\left(w_{, r}, \eta\right)
$$

where $\eta=w / r$.
Then, (8) takes the form

$$
\begin{aligned}
w_{, t t}= & \frac{1}{r^{2}}\left[\left(\frac{\partial \psi}{\partial w_{, r}}+\mu_{0} w_{, r t}\right) r^{2}\right]_{, r} \\
& -\frac{1}{r}\left(\frac{\partial \psi}{\partial \eta}+\mu_{0} \frac{w_{, t}}{r}\right),
\end{aligned}
$$

and in view of (3), we have the initial conditions

$$
\left.w\right|_{t=0}=w_{0},\left.\quad w_{, t}\right|_{t=0}=w_{1}
$$

and in view of (2) and (9), the boundary condition

$$
\left.\left(\frac{\partial \psi}{\partial w_{, r}}+\mu_{0} w_{, r t}\right)\right|_{S_{R}}=0
$$

where $S_{R}=\partial B_{R}$.

Global existence of regular solutions to problems (11) and (12) with the Dirichlet boundary condition

$$
\left.w\right|_{S_{R}}=0
$$

is proved in [13]. To formulate the main results of this paper we need the following.

Assumptions. Let us introduce the notation $\vartheta=w_{, r}, \eta=w / r$. Assume that

(1) $\psi(\vartheta, \eta)=\psi_{1}(\vartheta)+\psi_{2}(\eta)$,

(2) there exist positive constants $\alpha_{1}, \alpha_{2}, \beta_{1}, \beta_{2}$ such that

$$
\begin{array}{cc}
\frac{\partial^{2} \psi_{1}}{\partial \vartheta^{2}} \geq \alpha_{1}, & \frac{\partial^{2} \psi_{2}}{\partial \eta^{2}} \geq \alpha_{2}, \\
\left|\frac{\partial \psi_{1}}{\partial \vartheta}\right| \leq \beta_{1}|\vartheta|, & \left|\frac{\partial \psi_{2}}{\partial \eta}\right| \leq \beta_{2}|\eta|,
\end{array}
$$

(3) $w_{0} \in H_{\mu_{1}}^{2}(0, R), w_{1} \in H_{\mu_{1}}^{2}(0, R), \mu_{1} \in(2,1+\sqrt{5} / 2)$,

(4) $w_{1} \in L_{2, \mu_{2}}(0, R), w_{0} \in H_{\mu_{2}}^{1}(0, R), \mu_{2} \in(0,3 / 2), \psi_{1} \geq$ $\alpha_{1} \vartheta^{2}, \psi_{2} \geq \alpha_{2} \eta^{2},\left|\psi_{1, \vartheta}\right| \leq \beta_{1}|\vartheta|,\left|\psi_{2, \eta}\right| \leq \beta_{2}|\eta|$,

(5) $w_{1} \in L_{2, \mu_{3}}(0, R), w_{0} \in H_{\mu_{3}}^{1}(0, R), \mu_{3} \in(0,1 / 2)$.

Main Theorem. (1) Let Assumptions (1) - (3) hold. Then there exists a solution to problems (11)-(13) such that

$$
\begin{aligned}
& w_{, t t} \in B\left(0, T ; L_{2, \mu_{1}}(0, R)\right), \quad w_{, t} \in B\left(0, T ; H_{\mu_{1}}^{1}(0, R)\right), \\
& w \in B\left(0, T ; H_{\mu_{1}}^{2}(0, R)\right), \quad w_{, t t} \in L_{2}\left(0, T ; H_{\mu_{1}}^{1}(0, R)\right) .
\end{aligned}
$$

(2) Let Assumptions (1) and (4) hold, then

$$
\begin{gathered}
w_{, t} \in B\left(0, T ; L_{2, \mu_{2}}(0, R)\right) \cap L_{2}\left(0, T ; H_{\mu_{2}}^{1}(0, R)\right), \\
w \in B\left(0, T ; H_{\mu_{2}}^{1}(0, R)\right) .
\end{gathered}
$$


(3) Let Assumptions (1), (4), and (5) be satisfied. Then

$$
\begin{gathered}
w \in L_{\infty}((0, R) \times(0, T)), \quad w \in B\left(0, T ; C^{1 / 2-\mu_{3} / 2}(0, R)\right), \\
w \in L_{\beta}\left(0, T ; C^{(\beta-1) / \beta}(0, T)\right), \quad \beta \in\left(1, \frac{2}{2 \mu_{3}+1}\right), \\
w_{, t} \in L_{2}\left(0, T ; C^{1 / 2-\mu_{3} / 2}(0, R)\right) .
\end{gathered}
$$

Our paper is organized as follows. In Section 1, the formulation of the considered problem and the main results are presented. In Section 2, the notation is introduced. Mainly, we define the anisotropic Sobolev spaces with weights. Section 3 is devoted to the proof of energy-type estimates to solutions of problems (11)-(13).

In Section 4 the existence of the global solution for nonsmall data of problems (11)-(13) is proved.

\section{Notation and Auxiliary Results}

By $c$ we denote the generic constant which changes from formula to formula. By $c(\sigma), \sigma>0$, we denote a generic function which is always positive and increasing.

We replace forms of right-hand side (left-hand side) by the abbreviation r.h.s. (1.h.s.). We mark $w_{, t}=\partial_{t} w, w_{, r}=\partial_{r} w$, and so on.

By $B(I)$ we denote the space of bounded functions on the interval $I$.

By $H_{\mu}^{k}(0, R), \mu \in \mathbb{R}, k \in \mathbb{N} \cup\{0\}$, we denote a weighted Sobolev space with the finite norm

$$
\|u\|_{H_{\mu}^{k}(0, R)}=\left(\sum_{\alpha=0}^{k} \int_{0}^{R}\left|\partial_{r}^{\alpha} u\right|^{2} r^{2(\mu-k+|\alpha|)} d r\right)^{1 / 2}
$$

and $L_{2, \mu}(0, R)=H_{\mu}^{0}(0, R)$.

By $C^{\alpha}(I), \alpha \in(0,1)$ we denote the Hölder space with the finite norm

$$
\|u\|_{C^{\alpha}(I)}=\sup _{\tau \in I}|u(\tau)|+\sup _{\tau^{\prime}, \tau^{\prime \prime} \in I} \frac{\left|u\left(\tau^{\prime}\right)-u\left(\tau^{\prime \prime}\right)\right|}{\left|\tau^{\prime}-\tau^{\prime \prime}\right|^{\alpha}} .
$$

Next, we recall the Hardy inequality (see [14, Chapter 1, Section 2.15])

$$
\left|\frac{1}{p^{\prime}}-\mu\right|^{p} \int_{0}^{\infty} r^{p(\mu-1)}|f|^{p} d r \leq \int_{0}^{\infty} r^{p \mu}\left|f_{, r}\right|^{p} d r
$$

where $1 / p+1 / p^{\prime}=1, \mu \in \mathbb{R}$, and $\mu \neq 1 / p^{\prime}$.

The inequality holds also for functions with compact support. Assuming that supp $f \subset[0, R]$, we introduce $F(x)=\int_{x}^{\infty} f(y) d y$ and repeat the proof from [14, Chapter 1, Section 2.15].

From [15, Chapter 2, Section 3], we have the imbedding

$$
\begin{aligned}
& \|u\|_{L_{q}\left(0, T ; L_{p}(0, R)\right)} \\
& \quad \leq c\left(\|u\|_{L_{\infty}\left(0, T ; L_{2}(0, R)\right)}+\left\|\nabla_{r} u\right\|_{L_{2}\left(0, T ; L_{2}(0, R)\right)}\right),
\end{aligned}
$$

where $1 / p+2 / q \geq 1 / 2$.
Finally, we consider the problem

$$
u_{, t}-u_{, r r}=f,\left.\quad u\right|_{t=0}=u_{0},\left.\quad u_{, r}\right|_{r=R}=0 .
$$

To examine nonstationary problem (23), we need anisotropic weighted Sobolev spaces $V_{p, v}^{2,1}((0, R) \times(0, T)), p \in(1, \infty), v \in$ $\mathbb{R}$, of functions with the finite norm

$$
\begin{aligned}
& \|u\|_{V_{p, \nu}^{2,1}((0, R) \times(0, T))} \\
& \quad=\left(\sum_{\alpha+2 a \leq 2} \int_{0}^{T} \int_{0}^{R}\left|\partial_{r}^{\alpha} \partial_{t}^{a} u\right|^{p} r^{p(\nu-2+\alpha+2 a)} d r d t\right)^{1 / p} .
\end{aligned}
$$

Spaces $V_{p, \nu}^{2}(0, R)$ appropriate for elliptic problems were introduced in [16]. Moreover, we assume that $L_{p, v}(0, R)=$ $V_{p, v}^{0}(0, R)$.

The following result is valid.

Lemma 1. We assume that $f \in L_{p, v}((0, R) \times(0, T)), u_{0} \in$ $V_{p, v}^{2-2 / p}(0, R)$. Then there exists a solution to problem (23) such that $u \in V_{p, v}^{2,1}((0, R) \times(0, T))$ and

$$
\begin{aligned}
& \|u\|_{V_{p, \nu}^{2,1}((0, R) \times(0, T))} \\
& \quad \leq c\left(\|f\|_{L_{p, \nu}((0, R) \times(0, T))+\left\|u_{0}\right\|_{V_{p, \nu}^{2-2 / p}(0, R)}}\right) .
\end{aligned}
$$

In the case of elliptic equations, a similar result was proved in [17] for $p=2$ and in [16] for any $p \in(1, \infty)$.

The weighted Sobolev spaces with fractional derivatives are introduced in [16].

In the nonstationary case, Lemma 1 follows from the methods described in [18] in the case $p=2$. For the general $p$ Lemma 1 results from considerations in [18-20].

Finally, we introduce spaces used in this paper. We will define them by introducing finite norms.

Besov space $B_{p, v}^{l}\left(B_{R}\right), l \in \mathbb{R}_{+}, p \in(1, \infty), v \in \mathbb{R}$,

$$
\|u\|_{B_{p, v}^{l}\left(B_{R}\right)}=\|u\|_{B_{p, \nu}^{\circ l-l l]}\left(B_{R}\right)}+\|u\|_{V_{p, \nu}^{[l]}\left(B_{R}\right)},
$$

where $[l]$ is the integer part of $l$,

$$
\|u\|_{B_{p, \nu}^{* \alpha}\left(B_{R}\right)}=\left(\int_{0}^{R} \int_{0}^{R} \frac{\left|u\left(r_{1}\right) r_{1}^{\nu}-u\left(r_{2}\right) r_{2}^{\nu}\right|^{p}}{\left|r_{1}-r_{2}\right|^{1+p \alpha}} d r_{1} d r_{2}\right)^{1 / p}
$$

where $\alpha \in(0,1)$ and

$$
\|u\|_{V_{p, \nu}^{k}\left(B_{R}\right)}=\left(\sum_{\alpha \leq k} \int_{0}^{R}\left|\partial_{r}^{\alpha} u\right|^{p} r^{p(\nu-k+\alpha)} d r\right)^{1 / p}
$$

for $k$ integer. 
$B\left(B_{R} \times(0, T)\right)$ is the space of bounded functions.

$C^{\alpha}\left(\bar{B}_{R}\right), C^{\alpha, \beta}\left(\bar{B}_{R} \times[0, T]\right), \alpha, \beta \in(0,1)$, are the Hölder spaces with the finite norms

$$
\begin{aligned}
\|u\|_{C^{\alpha}\left(\bar{B}_{R}\right)}=\|u\|_{B\left(\bar{B}_{R}\right)}+\sup _{r_{1}, r_{2} \in \bar{B}_{R}} \frac{\left|u\left(r_{1}\right)-u\left(r_{2}\right)\right|}{\left|r_{1}-r_{2}\right|^{\alpha}}, & \\
\|u\|_{C^{\alpha, \beta}\left(\bar{B}_{R} \times[0, T]\right)}= & \|u\|_{B\left(\bar{B}_{R} \times[0, T]\right)} \\
& +\sup _{t \in[0, T]} \sup _{r_{1}, r_{2} \in \bar{B}_{R}} \frac{\left|u\left(r_{1}, t\right)-u\left(r_{2}, t\right)\right|}{\left|r_{1}-r_{2}\right|^{\alpha}} \\
& +\sup _{r \in \bar{B}_{R} t^{\prime}, t^{\prime \prime} \in[0, T]} \sup _{\left|t^{\prime}-t^{\prime \prime}\right|^{\beta}}
\end{aligned}
$$

where $\bar{B}_{R}$ is the closure of $B_{R}$.

By $L_{q}^{l}\left(0, T ; W_{p}^{k}\left(B_{R}\right)\right), l, k \in \mathbb{N} \cup\{0\}, p, q \in[1, \infty]$, we denote a space of functions with the finite norm

$$
\left\|\partial_{t}^{l} u\right\|_{L_{q}\left(0, T ; W_{p}^{k}\left(B_{R}\right)\right)} \cdot
$$

We introduce also the Sobolev spaces

$$
W_{p}^{k}\left(B_{R}\right)=\left\{u=u(x):\|u\|_{W_{p}^{k}\left(B_{R}\right)}=\sum_{l \leq k}\left\|\partial_{r}^{l} u\right\|_{L_{p}\left(B_{R}\right)}<\infty\right\},
$$

where $k \in \mathbb{Z}_{+} \cup\{0\}, p \in[1, \infty]$,

$$
\begin{aligned}
W_{p}^{k, k / 2}\left(B_{R}^{T}\right)=\{u & =u(r, t):\|u\|_{W_{p}^{k, k / 2}\left(B_{R}^{T}\right)} \\
& \left.=\sum_{l+2 a \leq k}\left\|\partial_{r}^{l} \partial_{t}^{a} u\right\|_{L_{p}\left(B_{R}^{T}\right)}<\infty\right\},
\end{aligned}
$$

where $k, k / 2 \in \mathbb{Z}_{+} \cup\{0\}, p \in[1, \infty], B_{R}^{T}=B_{R} \times(0, T), B_{R}=$ $\left\{r \in \mathbb{R}_{+}: r<R\right\}$,

$$
\begin{array}{r}
W_{p}^{k}(0, T)=\left\{u=u(t):\|u\|_{W_{p}^{k}(0, T)}\right. \\
\left.=\sum_{l \leq k}\left\|\partial_{t}^{l} u\right\|_{L_{p}(0, T)}<\infty\right\},
\end{array}
$$

$k \in \mathbb{Z}_{+} \cup\{0\}, p \in[0,1]$. For $k=0$, we have $W_{p}^{0}\left(B_{R}\right)=L_{p}\left(B_{R}\right)$, $W_{p}^{0,0}\left(B_{R}^{T}\right)=L_{p}\left(B_{R}^{T}\right), W_{p}^{0}(0, T)=L_{p}(0, T), B_{p}^{\alpha}\left(B_{R}\right)=B_{p, p}^{\alpha}\left(B_{R}\right)$ is the Besov space introduced in [14, Chapter 4, Section 18] and $W_{p}^{\alpha}\left(B_{R}\right)$ is the Sobolev-Slobodetsky space, where $\alpha \notin$ $\mathbb{Z}_{+}, p \in[1, \infty]$.

Moreover,

$$
V_{p, 0}^{k}\left(B_{R}\right) \subset W_{p}^{k}\left(B_{R}\right), \quad V_{p, 0}^{k, k / 2}\left(B_{R}^{T}\right) \subset W_{p}^{k, k / 2}\left(B_{R}^{T}\right) .
$$

Finally $H^{k}\left(B_{R}\right)=W_{2}^{k}\left(B_{R}\right), H^{k, k / 2}\left(B_{R}^{T}\right)=W_{2}^{k, k / 2}\left(B_{R}^{T}\right)$, and so on.

\section{Estimates}

To prove the Main Theorem we have to recall some estimates proved in [13].

Lemma 2 (see [13, Lemma 3.1]). We consider problems (11)(13). Assume that $w_{0}, w_{1}$ are initial data defined by (12). Let $c_{0}$ be a constant such that

$$
\int_{B_{R}}\left[\frac{1}{2} w_{1}^{2}+\psi\left(w_{0, r}, \frac{w_{0}}{r}\right)\right] r^{2} d r \equiv c_{0}^{2}<\infty,
$$

where $\psi$ is a positive function differentiable with respect to its arguments introduced by (10).

Then, solutions to problems (11)-(13) satisfy the estimate

$$
\begin{gathered}
\int_{B_{R}}\left[\frac{1}{2} w_{, t}^{2}+\psi\left(w_{, r}, \frac{w}{r}\right)\right] r^{2} d r+\int_{B_{R}^{t}}\left(w_{, r t}^{2}+\frac{w_{, t}^{2}}{r^{2}}\right) r^{2} d r d t^{\prime} \\
=\int_{B_{R}}\left[\frac{1}{2} w_{1}^{2}+\psi\left(w_{0, r}, \frac{w_{0}}{r}\right)\right] r^{2} d r=c_{0}^{2}, \text { for any } t>0 .
\end{gathered}
$$

Next, we need the following.

Lemma 3 (see [13, Lemma 3.2]). We consider problems (11)(13). Let $\vartheta=w_{, r}, \eta=w / r$. Assume that $\psi(\vartheta, \eta)=\psi_{1}(\vartheta)+\psi_{2}(\eta)$. Assume existence of positive constants $\alpha_{1}, \alpha_{2}, \beta_{1}$, and $\beta_{2}$ such that

$$
\begin{aligned}
\psi_{1}(\vartheta) \geq \alpha_{1} \vartheta^{2}, & \psi_{2}(\eta) \geq \alpha_{2} \eta^{2}, \\
\left|\frac{\partial \psi_{1}}{\partial \vartheta}\right| \leq \beta_{1}|\vartheta|, & \left|\frac{\partial \psi_{2}}{\partial \eta}\right| \leq \beta_{2}|\eta| .
\end{aligned}
$$

Finally, assume that

$$
w_{1} \in L_{2, \mu}(0, R), \quad w_{0} \in H_{\mu}^{1}(0, R), \quad \mu \in\left(0, \frac{3}{2}\right) .
$$

Then solutions to problems (11)-(13) satisfy the following estimate:

$$
\begin{aligned}
& \left\|w_{, t}\right\|_{L_{\infty}\left(0, t ; L_{2, \mu}\left(B_{R}\right)\right)}+\|w\|_{L_{\infty}\left(0, t ; H_{\mu}^{1}\left(B_{R}\right)\right)}+\left\|w_{, t}\right\|_{L_{2}\left(0, t ; H_{\mu}^{1}\left(B_{R}\right)\right)} \\
& \quad \leq c(R, \mu, t)\left(\left\|w_{1}\right\|_{L_{2, \mu}\left(B_{R}\right)}+\left\|w_{0}\right\|_{H_{\mu}^{1}\left(B_{R}\right)}\right) \\
& \quad \leq c_{1}(R, \mu, T)
\end{aligned}
$$

where $\mu \in(0,3 / 2), t \leq T$.

Continuing, we have the following.

Lemma 4 (see [13, Lemma 3.3]). We consider problems (11)(13). Assume that there exist positive constants $\bar{\alpha}_{1}, \bar{\alpha}_{2}, \bar{\beta}_{1}, \bar{c}_{1}$, and $\bar{c}_{2}$ such that

$$
\begin{gathered}
\psi_{1,99} \geq \bar{\alpha}_{1}, \quad \psi_{2, \eta \eta} \geq \bar{\alpha}_{2}, \quad\left|\psi_{1, \vartheta 9}\right| \leq \bar{\beta}_{1}, \\
\left|\partial_{\vartheta}^{3} \psi_{1}\right| \leq \bar{c}_{1}, \quad\left|\partial_{\eta}^{3} \psi_{2}\right| \leq \bar{c}_{2} .
\end{gathered}
$$


Assume also that

$$
\begin{gathered}
\eta_{, t}, \vartheta_{, t} \in L_{3}\left(0, T ; L_{3,2 / 3 \mu}\left(B_{R}\right)\right), \quad w_{1} \in H_{\mu}^{1}\left(B_{R}\right), \\
w_{, t t}(0) \in L_{2, \mu}\left(B_{R}\right),
\end{gathered}
$$

where

$$
\begin{aligned}
w_{, t t}(0)= & \frac{1}{r^{2}}\left[\left(\psi_{, 9}\left(w_{0, r}, \frac{w_{0}}{r}\right)+\mu_{0} w_{1, r}\right) r^{2}\right]_{, r} \\
& -\frac{1}{r}\left[\psi_{, \eta}\left(w_{0, r}, \frac{w_{0}}{r}\right)+\frac{w_{1}}{r}\right] .
\end{aligned}
$$

Then solutions to (11)-(13) satisfy the following inequality:

$$
\begin{aligned}
& \left\|w_{, t t}\right\|_{L_{\infty}\left(0, t ; L_{2, \mu}\left(B_{R}\right)\right)}+\left\|w_{, t}\right\|_{L_{\infty}\left(0, t ; H_{\mu}^{1}\left(B_{R}\right)\right)} \\
& \quad+\mu_{0}\left\|w_{, t t}\right\|_{L_{2}\left(0, t ; H_{\mu}^{1}\left(B_{R}\right)\right)} \\
& \leq c(t)\left(\left\|\vartheta_{, t}\right\|_{L_{3}\left(0, t ; L_{3,2 / 3 \mu}\left(B_{R}\right)\right)}^{3 / 2}+\left\|\eta_{, t}\right\|_{L_{3}\left(0, t ; L_{3,2 / 3 \mu}\left(B_{R}\right)\right)}^{3 / 2}\right)+c(t) c_{2},
\end{aligned}
$$

where $\mu \in(0,1+(1 / 2) \sqrt{5}), t \leq T$ and

$$
c_{2}=\left\|w_{, t t}(0)\right\|_{L_{2, \mu}\left(B_{R}\right)}+\left\|w_{, t}(0)\right\|_{H_{\mu}^{1}\left(B_{R}\right)} .
$$

Remark 5. In general constants $\alpha_{i}$ and $\bar{\alpha}_{i}, i=1,2$, are different. Let us consider the example

$$
\psi_{1}(\vartheta)=\alpha_{1} \vartheta^{2}+\frac{a}{\sqrt{1+\vartheta^{2}}}
$$

where $a$ is a positive constant. Then we have

$$
\psi_{1}(\vartheta) \geq \alpha_{1} \vartheta^{2}
$$

Continuing, we derive

$$
\psi_{1,99}=2 \alpha_{1}+\frac{a\left(2 \vartheta^{2}-1\right)}{\left(1+\vartheta^{2}\right)^{5 / 2}}
$$

For $\vartheta^{2} \geq 1 / 2$, we obtain $\psi_{1,99} \geq 2 \alpha_{1}$. But for $\vartheta^{2}<1 / 2$, we have

$$
\begin{aligned}
\psi_{1,99} & \geq 2 \alpha_{1}-\frac{a}{\left(1+\vartheta^{2}\right)^{5 / 2}} \\
& \geq 2 \alpha_{1}-a \equiv \bar{\alpha}_{1} .
\end{aligned}
$$

It is clear that many examples can be invented.

We need to obtain an estimate from (44). For this purpose, we examine the last two norms on the r.h.s. of (44). We express them in the form

$$
\left(\int_{0}^{T} \int_{B_{R}}\left|w_{, r t}\right|^{3} r^{2 \mu} d r d t\right)^{1 / 2}+\left(\int_{0}^{T} \int_{B_{R}}\left|\frac{w_{, t}}{r}\right|^{3} r^{2 \mu} d r d t\right)^{1 / 2} \equiv I .
$$

Hence, by the Hardy inequality (see [14, Chapter 1, Section 2.15]), the above expression is bounded by

$$
I \leq c\left(\int_{0}^{T} \int_{B_{R}}\left|w_{, r t}\right|^{3} r^{2 \mu} d r d t\right)^{1 / 2} \equiv I_{1} .
$$

To estimate $I_{1}$, we need the Pego transformation

$$
\begin{gathered}
p(r, t)=\int_{0}^{r} w_{, t}\left(r^{\prime}, t\right) r^{\prime 2} d r^{\prime}, \\
q(r, t)=\mu_{0} w_{, r}(r, t) r^{2}-p(r, t) .
\end{gathered}
$$

Hence,

$$
w_{, r t} r^{2}=\frac{1}{\mu_{0}}\left(q_{, t}+p_{, t}\right)
$$

To calculate the above expression we need problems for $p$ and $q$. From Lemma 3.4 from [13], we have the following problems for $p$ and $q$ :

$$
\begin{gathered}
p_{, t}-\mu_{0} p_{, r r}=\psi_{, 9} r^{2}-\int_{0}^{r}\left(\psi_{, \eta}+\mu_{0} \frac{w_{, t}}{r}\right) r d r \\
-2 \mu_{0} w_{, t} r, \\
\left.p\right|_{t=0}=\int_{0}^{r} w_{1}(r) r^{2} d r \equiv p_{0}(r),\left.\quad p_{, r}\right|_{r=R}=w_{, t}(R, t) R^{2}
\end{gathered}
$$

$$
\begin{gathered}
q_{, t}=-\psi_{, 9} r^{2}+\int_{0}^{r}\left(\psi_{, \eta}+\mu_{0} \frac{w_{, t}}{r}\right) r d r, \\
\left.q\right|_{t=0}=\mu_{0} w_{0, r} r^{2} \\
-\int_{0}^{r} w_{1}(r) r^{2} d r \equiv q_{0}(r) .
\end{gathered}
$$

Lemma 6. Let the assumptions of Lemma 3 be satisfied. Let $w_{, r} \in L_{\sigma, 2-\nu}\left(B_{R}^{T}\right), w_{, t}, \partial_{t}^{2-1 / \sigma} w \in L_{\sigma}\left(0, T ; L_{2,1}\left(B_{R}\right)\right), \partial_{t}^{2-1 / \sigma}$ means the fractional derivative. Then solutions to problems (54) and (55) satisfy

$$
\begin{gathered}
\|p\|_{W_{\sigma,-\nu}^{2,1}\left(B_{R}^{T}\right)} \leq c\left\|w_{, r}\right\|_{L_{\sigma, 2-\nu}\left(B_{R}^{T}\right)}+c\left\|w_{, t} r^{2}\right\|_{W_{\sigma}^{1,1 / 2}\left(B_{R, R_{0}}^{T}\right)}+c c_{1}, \\
\left\|q_{, t}\right\|_{L_{\sigma,-\nu}\left(B_{R}^{T}\right)} \leq c\left\|w_{, r}\right\|_{L_{\sigma, 2-\nu}\left(B_{R}^{T}\right)}+c c_{1}
\end{gathered}
$$

where $c_{1}$ is introduced in (40), $B_{R, R_{0}}=\left\{r: 0<R_{0}<r<R\right\}$, $\nu<1 / \sigma+3 / 2-\mu, v<1, \sigma \in(1,6]$, and $\mu \in(0,3 / 2)$ is introduced in Lemma 3.

Proof. For solutions to problem (54), we have (see Lemma 1),

$$
\begin{gathered}
\|p\|_{W_{\sigma,-\nu}^{2,1}\left(B_{R}^{T}\right)} \\
\leq c\left(\left\|w_{, r} r^{2}\right\|_{L_{\sigma,-\nu}\left(B_{R}^{T}\right)}+\left\|\int_{0}^{r}\left(|w|+\left|w_{, t}\right|\right) d r\right\|_{L_{\sigma,-\nu}\left(B_{R}^{T}\right)}\right. \\
+\left\|w_{, t} r\right\|_{L_{\sigma,-\nu}\left(B_{R}^{T}\right)}+\left\|p_{0}\right\|_{W_{\sigma,-\nu}^{2-2 / \sigma}\left(B_{R}\right)} \\
\left.+\left\|w_{, t}(R, t) R^{2}\right\|_{W_{\sigma,-\nu}^{1-1 / \sigma, 1 / 2-1 / 2 \sigma}\left(S_{R}^{T}\right)}\right) .
\end{gathered}
$$

Now, we examine the terms from the r.h.s. of (58). 
The first norm on the r.h.s. of (58) equals

$$
\left(\int_{B_{R}^{T}}\left|w_{, r}\right|^{\sigma} r^{(2-v) \sigma} d r d t\right)^{1 / \sigma} .
$$

Applying the Hölder inequality, the second integral on the r.h.s. of (58) is bounded by

$$
\begin{aligned}
\left(\int_{B_{R}^{T}} \mid\right. & \left(\int_{0}^{r} r^{-2 \nu_{1}} d r\right)^{1 / 2} \\
& \left.\times\left.\left(\int_{0}^{r}\left(w^{2}+w_{, t}^{2}\right) r^{2 \nu_{1}} d r\right)^{1 / 2}\right|^{\sigma} r^{-v \sigma} d r d t\right)^{1 / \sigma} \leq c c_{1},
\end{aligned}
$$

where the last inequality holds in virtue of Lemma 3 and under the assumption

$$
v_{1}+v<\frac{1}{\sigma}+\frac{1}{2}, \quad v_{1}=\mu-1,
$$

where $\mu \in(0,3 / 2)$ is introduced in Lemma 3. Hence,

$$
\mu+v<\frac{1}{\sigma}+\frac{3}{2}
$$

The third integral on the r.h.s. of (58) we express in the form

$$
\left(\int_{B_{R}^{T}}\left|w_{, t}\right|^{\sigma} r^{(1-v) \sigma} d r d t\right)^{1 / \sigma} \equiv J_{1} .
$$

Assuming $\nu<1$, setting $\mu=1-\nu>0$, and recalling imbedding (22) and Lemma 3, we obtain for $\sigma \leq 6$ the estimate

$$
\begin{aligned}
J_{1} \leq & \operatorname{csup}_{t}\left(\int_{B_{R}}\left|\left(w r^{\mu}\right)_{, t}\right|^{2} d r\right)^{1 / 2} \\
& +c\left(\int_{B_{R}^{T}}\left|\partial_{r}\left(w r^{\mu}\right)_{, t}\right|^{2} d r d t\right)^{1 / 2} \leq c c_{1} .
\end{aligned}
$$

Finally, the last term on the r.h.s. of (58) is bounded by

$$
\left\|w_{, t} r^{2}\right\|_{W_{\sigma}^{1-1 / \sigma, 1 / 2-1 / 2 \sigma}\left(S_{R}^{T}\right)} \leq c\left\|w_{, t} r^{2}\right\|_{W_{\sigma}^{1,1 / 2}\left(B_{R, R_{0}}^{T}\right)} .
$$

Using the above estimates in the r.h.s. of (58) yields (56).

Repeating the considerations leading to (56) to problem (55) gives (57). This concludes the proof.

Using (56) and (57) in (53) gives

$$
\begin{aligned}
& \left\|w_{, r t}\right\|_{L_{\sigma, 2-\nu}\left(B_{R}^{T}\right)} \\
& \quad \leq c\left\|w_{, r}\right\|_{L_{\sigma, 2-\nu}\left(B_{R}^{T}\right)}+c(T)\left\|w_{, t} r^{2}\right\|_{W_{\sigma}^{1,1 / 2}\left(B_{R, R_{0}}^{T}\right)}+c c_{1} .
\end{aligned}
$$

Applying the equality

$$
w_{, r}(t)=\int_{0}^{t} w_{, r t^{\prime}} d t^{\prime}+w_{, r}(0)
$$

in the first term on the r.h.s. of (66) and using the Gronwall lemma, we get

$$
\left\|w_{, r t}\right\|_{L_{\sigma, 2-\nu}\left(B_{R}^{T}\right)} \leq c(T)\left\|w_{, t} r^{2}\right\|_{W_{\sigma}^{1,1 / 2}\left(B_{R, R_{0}}^{T}\right)}+c(T) c_{1} .
$$

Now, we have to estimate the norm on the r.h.s. of (68). For the purpose we need the following.

Lemma 7. Let $w$ be a solution to (11)-(13). Assume that $\psi=$ $\psi_{1}(9)+\psi_{2}(\eta)$ and (37), (38) are satisfied. Assume that $w_{1} \in$ $W_{\sigma}^{1-1 / \sigma}\left(B_{R}\right), w_{0} \in W_{\sigma}^{1}\left(B_{R, R_{0 / 2}}\right)$, where $\sigma \in(1, \infty), 0<R_{0}<R$. Then

$$
\begin{aligned}
& \left\|w_{, t} r^{2}\right\|_{W_{\sigma}^{1,1 / 2}\left(B_{R, R_{0}}^{T}\right)} \\
& \quad \leq c(T)\left(c_{0}+\left\|w_{0}\right\|_{W_{\sigma}^{1}\left(B_{R, R_{0} / 2}\right)}+\left\|w_{1}\right\|_{W_{\sigma}^{1-1 / \sigma}\left(B_{R}\right)}\right),
\end{aligned}
$$

where $c_{0}$ is introduced in (36).

Proof. Let us introduce a smooth function $\zeta=\zeta(r)$ such that $\zeta(r)=0$ for $r<R_{0} / 2$ and $\zeta(r)=1$ for $r>R_{0}$, where $R_{0}<R$.

First, we obtain a local estimate in $B_{R, R_{0}}$ for solutions to problems (11)-(13). Multiplying (11) by $\zeta^{2} w_{, t} r^{2}$ and integrating over $B_{R}$ yields

$$
\begin{gathered}
\int_{B_{R}} w_{, t t} \zeta^{2} w_{, t} r^{2} d r+\int_{B_{R}}\left(\psi_{\vartheta}+\mu_{0} w_{, r t}\right)\left(\zeta^{2} w_{, t}\right)_{, r} r^{2} d r \\
+\int_{B_{R}}\left(\psi_{, \eta}+\mu_{0} \eta_{, t}\right) \zeta^{2} w_{, t} r d r=0 .
\end{gathered}
$$

Introducing the notation

$$
\widetilde{w}=w \zeta
$$

we obtain

$$
\begin{aligned}
& \frac{1}{2} \frac{d}{d t} \int_{B_{R}} \widetilde{w}_{, t}^{2} r^{2} d r+\int_{B_{R}}\left(\psi_{, \vartheta} \vartheta_{, t}+\psi_{, \eta} \eta_{, t}\right) \zeta^{2} r^{2} d r \\
& \quad+2 \int_{B_{R}} \psi{ }_{, \vartheta} w_{, t} \zeta \zeta_{, r} r^{2} d r \\
& \quad+\mu_{0} \int_{B_{R}}\left[w_{, r t}\left(\zeta^{2} w_{, t}\right)_{, r}+\frac{\widetilde{w}_{, t}}{r^{2}}\right] r^{2} d r=0 .
\end{aligned}
$$

Continuing, we have

$$
\begin{aligned}
& \quad \frac{d}{d t}\left(\frac{1}{2} \int_{B_{R}} \widetilde{w}_{, t}^{2} r^{2} d r+\int_{B_{R}} \psi \zeta^{2} r^{2} d r\right) \\
& +\mu_{0} \int_{B_{R}}\left(w_{, r t}^{2} \zeta^{2}+\frac{\widetilde{w}_{, t}^{2}}{r^{2}}\right) r^{2} d r \\
& =-2 \mu_{0} \int_{B_{R}} w_{, r t} w_{, t} \zeta \zeta_{, r} r^{2} d r-2 \int_{B_{R}} \psi_{, \vartheta} w_{, t} \zeta \zeta_{, r} r^{2} d r .
\end{aligned}
$$


Applying the Hölder and the Young inequalities to the r.h.s. of the above equality and using (38) yields

$$
\begin{aligned}
& \quad \frac{d}{d t}\left(\frac{1}{2} \int_{B_{R}} \widetilde{w}_{, t}^{2} r^{2} d r+\int_{B_{R}} \psi \zeta^{2} r^{2} d r\right) \\
& +\frac{\mu_{0}}{2} \int_{B_{R}}\left(w_{, r t}^{2} \zeta^{2}+\frac{\widetilde{w}_{, t}^{2}}{r^{2}}\right) r^{2} d r \\
& \leq c \mu_{0} \int_{B_{R}} w_{, t}^{2} \zeta_{, r}^{2} r^{2} d r+c \int_{B_{R}} w_{, r}^{2} \zeta_{, r}^{2} r^{2} d r .
\end{aligned}
$$

Using that $w_{, r t}^{2} \zeta^{2}=\left(\widetilde{w}_{, r t}-w_{, t} \zeta_{, r}\right)^{2}$ in the above inequality implies

$$
\begin{aligned}
& \quad \frac{d}{d t}\left(\frac{1}{2} \int_{B_{R}} \widetilde{w}_{, t}^{2} r^{2} d r+\int_{B_{R}} \psi \zeta^{2} r^{2} d r\right) \\
& +\mu_{0} \int_{B_{R}}\left(\widetilde{w}_{, r t}^{2}+\frac{\widetilde{w}_{, t}^{2}}{r^{2}}\right) r^{2} d r \\
& \leq c \mu_{0} \int_{B_{R}} w_{, t}^{2} \zeta_{, r}^{2} r^{2} d r+c \int_{B_{R}} w_{, r}^{2} \zeta_{, r}^{2} r^{2} d r .
\end{aligned}
$$

Integrating the above inequality with respect to time and using (37), we have

$$
\begin{aligned}
& \quad \frac{1}{2} \int_{B_{R}} \widetilde{w}_{, t}^{2} r^{2} d r+\int_{B_{R}}\left(\alpha_{1} w_{, r}^{2} \zeta^{2}+\alpha_{2} \frac{\widetilde{w}^{2}}{r^{2}}\right) r^{2} d r \\
& +\mu_{0} \int_{B_{R}^{t}}\left(w_{, r t}^{2}+\frac{\widetilde{w}_{, t}^{2}}{r^{2}}\right) r^{2} d r d t^{\prime} \\
& \leq c \mu_{0} \int_{B_{R}^{t}} w_{, t}^{2} \zeta_{, r}^{2} r^{2} d r d t^{\prime}+c \int_{B_{R}^{t}} w_{, r}^{2} \zeta_{, r}^{2} r^{2} d r d t^{\prime} \\
& \quad+\frac{1}{2} \int_{B_{R}} \widetilde{w}_{1} r^{2} d r+\int_{B_{R}} \psi\left(w_{0, r}, \frac{w_{0}}{r}\right) r^{2} d r \\
& \equiv c \int_{B_{R}^{t}}\left(w_{, t}^{2}+w_{, r}^{2}\right) \zeta_{, r}^{2} r^{2} d r d t^{\prime}+c c_{0}^{2} .
\end{aligned}
$$

Since $\widetilde{w}_{, r}^{2} \leq 2\left(\left(w_{, r} \zeta\right)^{2}+w^{2} \zeta_{, r}^{2}\right)$, the above inequality yields

$$
\begin{gathered}
\frac{1}{2} \int_{B_{R}} \widetilde{w}_{, t}^{2} r^{2} d r+\int_{B_{R}}\left(\frac{1}{2} \alpha_{1} \widetilde{w}_{, r}^{2} r^{2}+\alpha_{2} \widetilde{w}^{2}\right) d r \\
+\mu_{0} \int_{B_{R}^{t}}\left(\widetilde{w}_{, r t}^{2} r^{2}+\widetilde{w}_{, t}^{2}\right) d r d t^{\prime} \\
\leq c \int_{B_{R}^{t}}\left(w_{, t}^{2}+w_{, r}^{2}+w^{2}\right) \zeta_{, r}^{2} r^{2} d r d t^{\prime}+c c_{0}^{2} \leq c c_{0}^{2},
\end{gathered}
$$

where (36) was used again.

Now, we introduce the following Pego transformation

$$
\begin{gathered}
\tilde{p}(r, t)=\int_{0}^{r} \widetilde{w}_{, t}\left(r^{\prime}, t\right) r^{\prime 2} d r^{\prime}, \\
\tilde{q}(r, t)=\mu_{0} \widetilde{w}_{, r} r^{2}-\widetilde{p}(r, t) .
\end{gathered}
$$

Moreover, we have the initial-boundary value problem for $\widetilde{p}$,

$$
\begin{aligned}
& \widetilde{p}_{, t}-\mu_{0} \widetilde{p}_{, r r}=\int_{0}^{r} \widetilde{w}_{, t t} r^{2} d r-\mu_{0}\left(\widetilde{w}_{, t} r^{2}\right)_{, r} \equiv I, \\
& \left.\widetilde{p}\right|_{t=0}=\int_{0}^{r} \widetilde{w}_{1} r^{2} d r, \quad \widetilde{p}(R, t)=\int_{0}^{R} \widetilde{w}_{, t} r^{2} d r .
\end{aligned}
$$

The nonhomogeneous Dirichlet boundary condition is not convenient so we introduce the new function

$$
\widetilde{p}^{\prime}=\widetilde{p}-\widetilde{p}(R, t)
$$

which is a solution to the problem

$$
\begin{gathered}
\widetilde{p}_{, t}^{\prime}-\mu_{0} \widetilde{p}_{, r r}^{\prime}=I-\int_{0}^{R} \widetilde{w}_{, t t}^{2} r^{2} d r \equiv I_{1}, \\
\left.\widetilde{p}^{\prime}\right|_{t=0}=\int_{0}^{r} \widetilde{w}_{1} r^{2} d r-\int_{0}^{R} \widetilde{w}_{1} r^{2} d r \equiv \widetilde{p}_{0}^{\prime}, \\
\left.\widetilde{p}^{\prime}\right|_{r=R}=0 .
\end{gathered}
$$

We have to calculate $I_{1}$. For this purpose, we need the expressions which follow from (11) multiplied by $\zeta$

$$
\begin{aligned}
\widetilde{w}_{, t t}= & \frac{1}{r^{2}}\left[\left(\psi_{, \vartheta} \zeta+\mu_{0} w_{, r t} \zeta\right) r^{2}\right]_{, r}-\left(\psi_{, \vartheta}+\mu_{0} w_{, r t}\right) \zeta_{, r} \\
& -\frac{1}{r}\left(\psi_{, \eta} \zeta+\mu_{0} \frac{\widetilde{w}_{, t}}{r}\right)
\end{aligned}
$$

and from (13) multiplied by $\zeta$

$$
\left.\left(\psi_{, \vartheta}+\mu_{0} w_{, r t}\right) \zeta\right|_{r=R}=0
$$

Using (82) and (83) in $I_{1}$ yields

$$
\begin{aligned}
I_{1}= & \int_{0}^{r}\left[\left(\psi_{, \vartheta} \zeta+\mu_{0} w_{, r t} \zeta\right) r^{2}\right]_{, r} d r \\
& -\int_{0}^{r}\left(\psi_{, \vartheta}+\mu_{0} w_{, r t}\right) \zeta_{, r} r^{2} d r \\
& -\int_{0}^{r}\left(\psi_{, \eta} \zeta r+\mu_{0} \widetilde{w}_{, t}\right) d r-\mu_{0}\left(\widetilde{w}_{, t} r^{2}\right)_{, r} \\
& -\int_{0}^{R}\left[\left(\psi_{, \vartheta} \zeta+\mu_{0} w_{, r t} \zeta\right) r^{2}\right]_{, r} d r \\
& +\int_{0}^{R}\left(\psi_{, \vartheta}+\mu_{0} w_{, r t}\right) \zeta_{, r} r^{2} d r \\
& +\int_{0}^{R}\left(\psi_{, \eta} \zeta r+\mu_{0} \widetilde{w}_{, t}\right) d r
\end{aligned}
$$




$$
\begin{aligned}
= & \psi, 9 \\
& \zeta r^{2}+\mu_{0} \widetilde{w}_{, r t} r^{2}-\mu_{0} w_{, t} \zeta_{, r} r^{2} \\
& +\int_{r}^{R}\left(\psi_{, \vartheta}+\mu_{0} w_{, r t}\right) \zeta_{, r} r^{2} d r \\
& +\int_{r}^{R}\left(\psi_{, \eta} \zeta+\mu_{0} \widetilde{w}_{, t}\right) d r-\mu_{0}\left(\widetilde{w}_{, r t} r^{2}+2 r \widetilde{w}_{, t}\right) \\
= & \psi, \zeta r^{2}-\mu_{0} w_{, t} \zeta_{, r} r^{2}-2 \mu_{0} r \widetilde{w}_{, t} \\
& +\int_{r}^{R}\left(\psi_{, 9} \zeta_{, r} r^{2}+\psi_{, \eta} \zeta r+\mu_{0} \widetilde{w}_{, t}\right) d r \\
& -\mu_{0} \int_{r}^{R} w_{, t}\left(\zeta_{, r} r^{2}\right)_{, r} d r .
\end{aligned}
$$

For solutions to problem (81), we have

$$
\left\|\tilde{p}^{\prime}\right\|_{W_{\sigma}^{2,1}\left(B_{R}^{T}\right)} \leq c\left(\left\|I_{1}\right\|_{L_{\sigma}\left(B_{R}^{T}\right)}+\left\|\tilde{p}_{0}^{\prime}\right\|_{W_{\sigma}^{2-2 / \sigma}\left(B_{R}\right)}\right) .
$$

Hence,

$\|\tilde{p}\|_{W_{\sigma}^{2,1}\left(B_{R}^{T}\right)}$

$$
\leq c\left(\left\|I_{1}\right\|_{L_{\sigma}\left(B_{R}^{T}\right)}+\left\|\tilde{p}_{, t}(R, t)\right\|_{L_{\sigma}\left(B_{R}^{T}\right)}+\left\|\tilde{p}_{0}^{\prime}\right\|_{W_{\sigma}^{2-2 / \sigma}\left(B_{R}\right)}\right) .
$$

From the expression of $I_{1}$, we have

$$
\left\|I_{1}\right\|_{L_{\sigma}\left(B_{R}^{T}\right)}
$$

$$
\leq c\left(\left\|w_{, r}\right\|_{L_{\sigma}\left(B_{R, R_{0} / 2}^{T}\right)}+\left\|w_{, t}\right\|_{L_{\sigma}\left(B_{R, R_{0} / 2}^{T}\right)}+\|w\|_{L_{\sigma}\left(B_{R, R_{0} / 2}^{T}\right)}\right) .
$$

In view of (82) and (83), we obtain

$$
\begin{aligned}
\widetilde{p}_{, t}(R, t)= & \int_{0}^{R} \widetilde{w}_{, t t} r^{2} d r \\
= & \int_{0}^{R}\left(\psi_{, \vartheta}+\mu_{0} w_{, r t}\right) \zeta_{, r} r^{2} d r \\
& -\int_{0}^{R}\left(\psi_{, \eta} \zeta r+\mu_{0} \widetilde{w}_{, t}\right) d r .
\end{aligned}
$$

Hence,

$$
\begin{aligned}
& \left|\widetilde{p}_{, t}(R, t)\right| \\
& \leq\left|\int_{0}^{R}\left(\psi_{, \vartheta} \zeta_{, r} r^{2}+\psi_{, \eta} \zeta r+\mu_{0} \widetilde{w}_{, t}\right) d r\right| \\
& \quad+\left|\int_{0}^{R} \mu_{0} w_{, t}\left(\zeta_{, r} r^{2}\right)_{, r} d r\right| .
\end{aligned}
$$

Employing (38) yields

$$
\begin{aligned}
& \left\|\tilde{p}_{, t}(R, t)\right\|_{L_{\sigma}\left(B_{R}^{T}\right)} \\
& \quad \leq c\left(\left\|w_{, r}\right\|_{L_{\sigma}\left(B_{R, R_{0} / 2}^{T}\right)}+\left\|w_{, t}\right\|_{L_{\sigma}\left(B_{R, R_{0} / 2}^{T}\right)}+\|w\|_{L_{\sigma}\left(B_{R, R_{0} / 2}^{T}\right)}\right) .
\end{aligned}
$$

From the form of $\widetilde{p}_{0}^{\prime}\left(\right.$ see $\left.(81)_{2}\right)$, we derive

$$
\begin{aligned}
\left\|\widetilde{p}^{\prime}{ }_{0}\right\|_{W_{\sigma}^{2-2 / \sigma}\left(B_{R}\right)} & =\left\|\int_{0}^{r} \widetilde{w}_{1} r^{2} d r\right\|_{W_{\sigma}^{2-2 / \sigma}\left(B_{R}\right)} \\
& \leq\left\|\widetilde{w}_{1} r^{2}\right\|_{W_{\sigma}^{1-2 / \sigma}\left(B_{R}\right)} \leq c\left\|\widetilde{w}_{1}\right\|_{W_{\sigma}^{1-2 / \sigma}\left(B_{R}\right)} .
\end{aligned}
$$

Using (90) in (86) yields

$$
\begin{aligned}
\|\widetilde{p}\|_{W_{\sigma}^{2,1}\left(B_{R}^{T}\right)} \leq c( & \left\|w_{, r}\right\|_{L_{\sigma}\left(B_{R, R_{0} / 2}^{T}\right)}+\left\|w_{, t}\right\|_{L_{\sigma}\left(B_{R, R_{0} / 2}^{T}\right)} \\
& \left.+\|w\|_{L_{\sigma}\left(B_{R, R_{0} / 2}^{T}\right)}+\left\|w_{1}\right\|_{W_{\sigma}^{1-2 / \sigma}\left(B_{R, R_{0} / 2}^{T}\right)}\right) .
\end{aligned}
$$

Since $\widetilde{p}_{, r}=\widetilde{w}_{, t} r^{2}$ and that

$$
\left\|\widetilde{p}_{, r}\right\|_{W_{\sigma}^{1,1 / 2}\left(B_{R}^{T}\right)} \leq c\|\widetilde{p}\|_{W_{\sigma}^{2,1}\left(B_{R}^{T}\right)}
$$

we obtain from (92) the inequality

$$
\begin{aligned}
\left\|w_{, t} r^{2}\right\|_{W_{\sigma}^{1,1 / 2}\left(B_{R, R_{0} / 2}^{T}\right)} & \\
\leq c(T) & \left(\left\|w_{, r}\right\|_{L_{\sigma}\left(B_{R, R_{0} / 2}^{T}\right)}+\left\|w_{, t}\right\|_{L_{\sigma}\left(B_{R, R_{0} / 2}^{T}\right)}\right. \\
& \left.+\left\|w_{0}\right\|_{L_{\sigma}\left(B_{R, R_{0} / 2}\right)}+\left\|w_{1}\right\|_{W_{\sigma}^{1-2 / \sigma}\left(B_{R, R_{0} / 2}\right)}\right),
\end{aligned}
$$

where the relation $w(t)=\int_{0}^{t} w_{, t^{\prime}} d t^{\prime}+w_{0}$ was exploited.

Using the interpolation inequality

$$
\begin{aligned}
& \left\|w_{, t}\right\|_{L_{\sigma}\left(B_{R, R_{0} / 2}^{T}\right)} \\
& \quad \leq \varepsilon\left\|w_{, r t}\right\|_{L_{\sigma}\left(B_{R, R_{0} / 2}^{T}\right)}+c\left(\frac{1}{\varepsilon}\right)\left\|w_{, t}\right\|_{L_{2}\left(B_{R, R_{0} / 2}^{T}\right)}
\end{aligned}
$$

in (94), assuming that $\varepsilon$ is sufficiently small, and using (77), we obtain

$$
\begin{gathered}
\left\|w_{, t} r^{2}\right\|_{W_{\sigma}^{1,1 / 2}\left(B_{R, R_{0} / 2}^{T}\right)} \\
\leq c(T)\left(\left\|w_{, r}\right\|_{L_{\sigma}\left(B_{R, R_{0} / 2}^{T}\right)}+\left\|w_{0}\right\|_{L_{\sigma}\left(B_{R, R_{0} / 2}\right)}\right. \\
\left.+\left\|w_{1}\right\|_{W_{\sigma}^{1-2 / \sigma}\left(B_{R, R_{0} / 2}\right)}+c_{0}\right) .
\end{gathered}
$$

Employing

$$
w_{, r}(t)=\int_{0}^{t} w_{, r t^{\prime}} d t^{\prime}+w_{, r}(0)
$$

in (96) and applying the Gronwall lemma, we get

$$
\begin{aligned}
& \left\|w_{, t} r^{2}\right\|_{W_{\sigma}^{1,1 / 2}\left(B_{R, R_{0} / 2}^{T}\right)} \\
& \quad \leq c(T)\left(\left\|w_{0}\right\|_{W_{\sigma}^{1}\left(B_{R, R_{0} / 2}\right)}+\left\|w_{1}\right\|_{W_{\sigma}^{1-2 / \sigma}\left(B_{R, R_{0} / 2}\right)}+c_{0}\right) .
\end{aligned}
$$

From (98), we obtain (69). This concludes the proof. 
To derive an estimate for (44), we have to take into account Lemmas 6 and 7. The lemmas imply an estimate for the first two terms on the r.h.s. of (44). For this purpose, we need the following.

Lemma 8. Let $w$ be a solution to problems (11)-(13). Assume that there exist constants $\bar{\alpha}_{i}, \bar{c}_{i}, i=1,2, \bar{\beta}_{1}$ such that (41) holds, where $\psi=\psi_{1}(\vartheta)+\psi_{2}(\eta), \vartheta=w_{, r}, \eta=w / r$. Assume that $w_{, t t}(0) \in L_{2, \mu_{1}}\left(B_{R}\right), w_{0} \in W_{3}^{1}\left(B_{R, R_{0} / 2}\right) \cap H_{\mu_{2}}^{1}\left(B_{R}\right), w_{1} \in$ $H_{\mu_{1}}^{1}\left(B_{R}\right) \cap L_{2, \mu_{2}}\left(B_{R}\right) \cap W_{3}^{2 / 3}\left(B_{R, R_{0} / 2}\right), c_{0}$ is defined in (36),

$$
\begin{gathered}
c_{1}=\left\|w_{1}\right\|_{L_{2, \mu_{2}\left(B_{R}\right)}}+\left\|w_{0}\right\|_{H_{\mu_{2}}^{1}\left(B_{R}\right)}, \\
c_{2}=\left\|w_{, t t}(0)\right\|_{L_{2, \mu_{1}}\left(B_{R}\right)}+\left\|w_{1}\right\|_{H_{\mu_{1}}^{1}\left(B_{R}\right)}, \\
\mu_{1} \in\left(\frac{3}{2}, 1+\frac{1}{2} \sqrt{5}\right), \quad \mu_{2} \in\left(\frac{5}{6}, \frac{3}{2}\right) .
\end{gathered}
$$

Then the following estimate holds:

$$
\begin{gathered}
\left\|w_{, t t}\right\|_{L_{\infty}\left(0, t ; L_{2, \mu_{1}}\left(B_{R}\right)\right)}+\left\|w_{, t}\right\|_{L_{\infty}\left(0, t ; H_{\mu_{1}}^{1}\left(B_{R}\right)\right)}+\left\|w_{, t t}\right\|_{L_{2}\left(0, t ; H_{\mu_{1}}^{1}\left(B_{R}\right)\right)} \\
\leq c(t)\left[\left(c_{1}+c_{0}+\left\|w_{0}\right\|_{W_{3}^{1}\left(B_{R, R_{0} / 2}\right)}\right.\right. \\
\left.\left.+\left\|w_{1}\right\|_{W_{3}^{2 / 3}\left(B_{R, R_{0} / 2}\right)}\right)^{3 / 2}+c_{2}\right], \quad t \leq T .
\end{gathered}
$$

Proof. From (44) and (51), we have

$$
\begin{gathered}
\left\|w_{, t t}\right\|_{L_{\infty}\left(0, t ; L_{2, \mu_{1}}\left(B_{r}\right)\right)}+\left\|w_{, t}\right\|_{L_{\infty}\left(0, t ; H_{\mu_{1}}^{1}\left(B_{R}\right)\right)}+\left\|w_{, t t}\right\|_{L_{2}\left(0, t ; H_{\mu_{1}}^{1}\left(B_{R}\right)\right)} \\
\leq c(t)\left[\left(\int_{B_{R}^{T}}\left|w_{, r t}\right|^{3} r^{2 \mu_{1}} d r d t\right)^{1 / 2}+c_{2}\right]
\end{gathered}
$$

where $\mu_{1} \in(0,1+(1 / 2) \sqrt{5}), t \leq T, c_{2}=\left\|w_{, t t}(0)\right\|_{L_{2, \mu_{1}}\left(B_{r}\right)}+$ $\left\|w_{, t}(0)\right\|_{H_{\mu_{1}}^{1}\left(B_{R}\right)}$.

Inequality (68) takes the form

$$
\left\|w_{, r t}\right\|_{L_{\sigma, 2-\gamma}\left(B_{R}^{t}\right)} \leq c(t)\left(\left\|w_{, t} r^{2}\right\|_{W_{\sigma}^{1,1 / 2}\left(B_{R, R_{0}}^{t}\right)}+c_{1}\right),
$$

where $c_{1}=\left\|w_{1}\right\|_{L_{2, \mu_{2}}\left(B_{R}\right)}+\left\|w_{0}\right\|_{H_{\mu_{2}}^{1}\left(B_{R}\right)}, \mu_{2} \in(0,3 / 2)$.

Finally, (69) yields

$$
\begin{aligned}
& \left\|w_{,} r^{2}\right\|_{W_{\sigma}^{1,1 / 2}\left(B_{R, R_{0}}^{t}\right)} \\
& \quad \leq c(t)\left(c_{0}+\left\|w_{0}\right\|_{W_{\sigma}^{1}\left(B_{R, R_{0} / 2}\right)}+\left\|w_{1}\right\|_{W_{\sigma}^{1-1 / \sigma}\left(B_{R, R_{0} / 2}\right)}\right) .
\end{aligned}
$$

To apply (102) and (103) in the r.h.s. of (101), we assume $v=$ $2-(2 / 3) \mu_{1}, \sigma=3$. In view of the assumptions of Lemma 6 , we have that $\nu<1$ so $2-(2 / 3) \mu_{1}<1$. This implies that $\mu_{1}>3 / 2$. Hence, $\mu_{1} \in(3 / 2,1+(1 / 2) \sqrt{5})$. Since $v=2-(2 / 3) \mu_{1}$, assumptions of Lemma 6 imply that $\mu_{2}<1 / 3+3 / 2-v=$ $11 / 6-2+(2 / 3) \mu_{1}$, so $\mu_{2} \in(5 / 6,1 / 2+(1 / 3) \sqrt{5}) \cap(0,3 / 2)=$ $(5 / 6,3 / 2)$. Employing (102) and (103) in (101) yields (100). This concludes the proof.
Remark 9. In view of the expression of $w_{, t}(0)$ appeared in the assumptions of Lemma 4 we derive

$$
\left\|w_{, t t}(0)\right\|_{L_{2, \mu_{1}}\left(B_{R}\right)} \leq c\left(\left\|w_{0}\right\|_{H_{\mu_{1}}^{2}\left(B_{R}\right)}+\left\|w_{1}\right\|_{H_{\mu_{1}}^{2}\left(B_{R}\right)}\right) .
$$

Since $\mu_{1}-2 \in(-1 / 2,(1 / 2) \sqrt{5}-1)$ can be chosen positive, we see that all weights in the norms $\left\|w_{0}\right\|_{H_{\mu_{1}}^{2}\left(B_{R}\right)},\left\|w_{1}\right\|_{H_{\mu_{1}}^{2}\left(B_{R}\right)}$ can be chosen as power functions with positive exponents.

Assuming that the r.h.s. of (104) is finite assumptions (99) can be replaced by

$$
\begin{gathered}
w_{1}, w_{0} \in H_{\mu_{1}}^{2}\left(B_{R}\right), \quad \mu_{1} \in\left(\frac{3}{2}, 1+\frac{\sqrt{5}}{2}\right), \\
\mu_{2} \geq \mu_{1}-1, \quad \mu_{2} \in\left(\frac{5}{6}, \frac{3}{2}\right) .
\end{gathered}
$$

Up to now there is no estimate for $w_{r r}$. Hence, to get the space regularity, we need the following.

Lemma 10. Let (105) hold. Let

$$
\psi_{, 99} \geq \alpha_{1}, \quad|\psi, 9| \leq c|\vartheta|, \quad|\psi, \eta| \leq c|\eta| .
$$

Then solutions to problems (11)-(13) satisfy

$$
\int_{0}^{T} \int_{0}^{R} w_{, r r}^{2} r^{2 \mu} d r d t+\int_{0}^{R} w_{, r r}^{2} r^{2 \mu} d r \leq \varphi\left(c_{0}, c_{1}, c_{3}, T\right),
$$

where $2<\mu<(1 / 3)(5+\sqrt{5}), c_{0}$ is from (36), $c_{1}$ from (40) and

$$
c_{3}=\left\|w_{0}\right\|_{H_{\mu}^{2}\left(B_{R}\right)}+\left\|w_{1}\right\|_{H_{\mu}^{2}\left(B_{R}\right)} .
$$

Proof. Multiplying (11) by $w_{, r r} r^{2 \mu}$ and integrating over $B_{R}$, we obtain

$$
\begin{aligned}
\int_{0}^{R} w_{, t} w_{, r r} r^{2 \mu} d r= & \int_{0}^{R} \frac{1}{r^{2}}\left[\left(\psi_{, \vartheta}+\mu_{0} w_{, r t}\right) r^{2}\right]_{, r} w_{, r r} r^{2 \mu} d r \\
& -\int_{0}^{R}\left(\frac{1}{r} \psi_{, \eta}+\mu_{0} \frac{w_{, t}}{r}\right) w_{, r r} r^{2 \mu} d r .
\end{aligned}
$$

Continuing, we have

$$
\begin{aligned}
\int_{0}^{R}\left(\psi_{, 99} w_{, r r}^{2}+\mu_{0} w_{, r r t} w_{, r r}\right) r^{2 \mu} d r \\
=\int_{0}^{R} w_{, t t} w_{, r r} r^{2 \mu} d r \\
\quad-2 \int_{0}^{R}\left(\psi_{, \vartheta}+\mu_{0} w_{, r t}\right) w_{, r r} r^{2 \mu-1} d r \\
\quad+\int_{0}^{R}\left(\psi_{, \eta}+\mu_{0} w_{, t}\right) w_{, r r} r^{2 \mu-1} d r .
\end{aligned}
$$


Using (106), we obtain

$$
\begin{aligned}
& r l \alpha_{1} \int_{0}^{R} w_{, r r}^{2} r^{2 \mu} d r+\frac{\mu_{0}}{2} \frac{d}{d t} \int_{0}^{R} w_{, r r}^{2} r^{2 \mu} d r \\
& \leq \varepsilon \int_{0}^{R} w_{, r r}^{2} r^{2 \mu} d r+c\left(\frac{1}{\varepsilon}\right) \int_{0}^{R} w_{, t t}^{2} r^{2 \mu} d r \\
& \quad+c \int_{0}^{R}\left(w_{, r}^{2}+w_{, r t}^{2}+w_{, t}^{2}+w^{2} r^{-2}\right) r^{2 \mu-2} d r .
\end{aligned}
$$

Integrating the inequality with respect to time, assuming that $\varepsilon$ is sufficiently small, using that $\mu=1+\nu, v \in(0,3 / 2)$, and employing (40) with $\mu=v$ and (102), (103) with $\sigma=2, \nu=$ $2-(2 / 3) \mu_{1}, \mu=(2 / 3) \mu_{1}+1$, we obtain (101). Hence, $\mu \in$ $(1,5 / 2) \cap(2,5 / 3+\sqrt{5} / 3)=(2,(1 / 3)(5+\sqrt{5}))$. This concludes the proof.

Finally, we recall some local properties of solutions to problems (11)-(13) which are proved in Lemma 3.11 in [13].

Lemma 11. Let the assumptions of Lemma 3 be satisfied. Then

$$
\begin{gathered}
|w(r, t)| \leq \frac{R^{1 / 2-\mu}}{\sqrt{1-2 \mu}} c_{1}, \quad \mu \in\left(0, \frac{1}{2}\right) \\
\left\|w_{, t}\right\|_{L_{2}\left(0, T ; C^{1 / 2-\mu / 2}\left(B_{R}\right)\right)} \leq c c_{1}, \quad \mu \in\left(0, \frac{1}{2}\right) \\
\|w\|_{B\left(0, T ; C^{1 / 2-\mu / 2}\left(B_{R}\right)\right)} \leq c c_{1}, \quad \mu \in\left(0, \frac{1}{2}\right) \\
\|w\|_{L_{\beta}\left(0, R ; C^{(\beta-1) / \beta}(0, T)\right)} \leq c c_{1}, \quad 1<\beta<\frac{2}{2 \mu+1}, \mu \in\left(0, \frac{1}{2}\right) .
\end{gathered}
$$

\section{Existence}

We prove the existence of solutions to problems (11)-(13) by the following method of successive approximations:

$$
\begin{aligned}
& w_{, t t}^{(n+1)}-\mu_{0} \frac{1}{r^{2}}\left(w_{, r t}^{(n+1)} r^{2}\right)_{, r}+\mu_{0} \frac{1}{r^{2}} w_{, t}^{(n+1)} \\
& =\frac{1}{r^{2}}\left(\psi_{, 9^{(n)}} r^{2}\right)_{, r}-\frac{1}{r} \psi_{, \eta^{(n)}}, \\
& \left.w^{(n+1)}\right|_{t=0}=w_{0},\left.\quad w_{, t}^{(n+1)}\right|_{t=0}=w_{1}, \\
& \left.w_{, r}^{(n+1)}\right|_{r=R}=0, \quad n \in \mathbb{N} \cup\{0\},
\end{aligned}
$$

where $\vartheta^{(n)}=w_{r}^{(n)}, \eta^{(n)}=w^{(n)} / r$, and we assume that the zero approximation is defined by

$$
w^{(0)}=w_{0}+t w_{1}
$$

The boundary condition (113) $)_{3}$ implies (13) because

$$
\left|\frac{\partial \psi}{\partial \vartheta}\right| \leq c|\vartheta|
$$

First, we examine the linear problem

$$
\begin{gathered}
u_{, t}-\mu_{0} u_{, r r}-\frac{2 \mu_{0} u_{, r}}{r}+\mu_{0} \frac{u}{r^{2}}=f \quad \text { in } B_{R}^{T}, \\
\left.u\right|_{t=0}=u_{1} \quad \text { in } B_{R}, \\
\left.u_{, r}\right|_{r=R}=0 \quad \text { on } S_{R} .
\end{gathered}
$$

Lemma 12. Assume that $f \in L_{2, \nu}\left(B_{R}^{T}\right), u_{1} \in H_{\nu}^{1}\left(B_{R}\right), v \in(2-$ $(1 / 2) \sqrt{5}, 2+(1 / 2) \sqrt{5})$. Then there exists a solution to problem (116) such that $u \in H_{v}^{2,1}\left(B_{R}^{T}\right)$ and the estimate holds

$$
\|u\|_{H_{v}^{2,1}\left(B_{R}^{T}\right)} \leq c\left(\|f\|_{L_{2, v}\left(B_{R}^{T}\right)}+\left\|u_{1}\right\|_{H_{\nu}^{1}\left(B_{R}\right)}\right) .
$$

Proof. The existence can be proved by the Galerkin method. Therefore, we shall restrict the proof to show only estimate (117).

Multiplying (116) by $u r^{2 \mu}$ and integrating the result over $B_{R}$ yields

$$
\begin{aligned}
& \frac{1}{2} \frac{d}{d t} \int_{B_{R}} u^{2} r^{2 \mu} d r+\mu_{0} \int_{B_{R}} u_{, r}^{2} r^{2 \mu} d r \\
& +2(\mu-1) \mu_{0} \int_{B_{R}} u_{, r} u r^{2 \mu-1} d r \\
& \quad+\mu_{0} \lim _{r \rightarrow 0}\left(u_{, r} u r^{2 \mu}\right)+\mu_{0} \int_{B_{R}} u^{2} r^{2 \mu-2} d r \\
& =\int_{B_{R}} f u r^{2 \mu} d r .
\end{aligned}
$$

Looking for solutions that $u_{r} u$ is finite at $r=0$, the last but one term on the l.h.s. of (118) vanishes for $\mu>0$. Vanishing of the term can be guaranteed by choosing an appropriate base functions.

Integrating by parts in the third term on the l.h.s. of (118) implies

$$
\begin{aligned}
& \frac{1}{2} \frac{d}{d t} \int_{B_{R}} u^{2} r^{2 \mu} d r+\mu_{0} \int_{B_{R}} u_{, r}^{2} r^{2 \mu} d r \\
& \quad+\mu_{0} \mu(3-2 \mu) \int_{B_{R}} u^{2} r^{2 \mu-2} d r+\left.(1-\mu) \mu_{0} u^{2} r^{2 \mu-1}\right|_{r=0} \\
& =(1-\mu) \mu_{0} u^{2}(R, t) R^{2 \mu-1}+\int_{B_{R}} f u r^{2 \mu} d r .
\end{aligned}
$$

For $\mu \in(0,1)$, the last term on the l.h.s. is positive so it can be omitted in the estimate implied by (119). Then we show the existence of solutions such that

$$
\int_{0}^{T} \int_{0}^{R} u^{2} r^{2 \mu-2} d r<\infty .
$$

Hence, $u \sim r^{\varepsilon}$ near $r=0$, where $\varepsilon>-\mu+1 / 2$. Then the last term on the 1.h.s. of (119) vanishes. For $\mu>1$, the last term on the 1.h.s. of (119) vanishes under the assumption that we are looking for solutions such that $u(r, t) \sim r^{\varepsilon}$ near $r=0$, $\varepsilon>-\mu+1 / 2$. 
Finally, we estimate the first term on the r.h.s. of (119). Let us recall the set

$$
B_{R, R_{0}}=\left\{r \in(0, R): 0<R_{0}<r<R\right\} .
$$

Then by imbedding, we get

$$
\begin{aligned}
|u(R, t)|^{2} & \leq \varepsilon \int_{B_{R, R_{0}}} u_{, r}^{2} d r+c\left(\frac{1}{\varepsilon}\right) \int_{B_{R, R_{0}}} u^{2} d r \\
& \leq \varepsilon^{\prime} \int_{B_{R}} u_{, r}^{2} r^{2 \mu} d r+c\left(\frac{1}{\varepsilon^{\prime}}\right) \int_{B_{R}} u^{2} r^{2 \mu} d r
\end{aligned}
$$

where $\varepsilon^{\prime}=\varepsilon / R_{0}^{2 \mu}$.

Using that $\varepsilon^{\prime}$ is sufficiently small, we obtain

$$
\begin{aligned}
& \frac{1}{2} \frac{d}{d t} \int_{B_{R}} u^{2} r^{2 \mu} d r+\mu_{0}^{\prime} \int_{B_{R}} u_{, r}^{2} r^{2 \mu} d r \\
& \quad+\mu_{0}^{\prime} \mu(3-2 \mu) \int_{B_{R}} u^{2} r^{2 \mu-2} d r \\
& \leq c \int_{B_{R}} u^{2} r^{2 \mu} d r+\left(\int_{B_{R}} f^{2} r^{2 \mu} d r\right)^{1 / 2}\left(\int_{B_{R}} u^{2} r^{2 \mu} d r\right)^{1 / 2} \\
& \leq c \int_{B_{R}} u^{2} r^{2 \mu} d r+c \int_{B_{R}} f^{2} r^{2 \mu} d r .
\end{aligned}
$$

Integrating (123) with respect to time, we get the estimate

$$
\begin{gathered}
\int_{B_{R}} u^{2} r^{2 \mu} d r+\mu_{0}^{\prime} \int_{B_{R}^{t}} u_{, r}^{2} r^{2 \mu} d r d t \\
+\mu_{0} \mu(3-2 \mu) \int_{B_{R}^{t}} u^{2} r^{2 \mu-2} d r d t \\
\leq e^{c t}\left(\int_{B_{R}^{t}} f^{2} r^{2 \mu} d r+\int_{B_{R}} u_{1}^{2} r^{2 \mu} d r\right) \equiv \alpha_{1}(t) .
\end{gathered}
$$

The above inequality implies the estimate for $\mu \in[0,3 / 2]$. To increase the interval, we use the Hardy inequality

$$
\left|\frac{1}{2}-\mu\right|^{1 / 2} \int_{0}^{R} u^{2} r^{2 \mu-2} d r \leq \int_{0}^{R} u_{, r}^{2} r^{2 \mu} d r .
$$

Combining (124) and (125), we see that (124) gives an estimate for $\mu$ satisfying the inequality

$$
\alpha\left|\frac{1}{2}-\mu\right|^{2}+\mu(3-2 \mu)>0, \quad \alpha=\frac{\mu_{0}^{\prime}}{\mu_{0}} .
$$

Solving (126), we see that $\mu \in((3-\alpha-\sqrt{9-4} \alpha) / 2(2-\alpha)$, (3$\alpha+\sqrt{9-4} \alpha) / 2(2-\alpha))$. Since $\alpha$ is very close to 1 , we can assume that

$$
\mu \in\left(1-\frac{1}{2} \sqrt{5}, 1+\frac{1}{2} \sqrt{5}\right) .
$$

Hence, $[0,3 / 2] \subset(1-(1 / 2) \sqrt{5}, 1+(1 / 2) \sqrt{5})$.
Finally, for $\mu$ satisfying (127), we have estimate (124). In the next step, we consider the parabolic problem

$$
\begin{aligned}
& u_{, t}-\mu_{0} u_{, r r}=2 \mu_{0} \frac{u_{, r}}{r}-\mu_{0} \frac{u}{r^{2}}+f, \\
& \left.u\right|_{t=0}=u_{1},\left.\quad u_{, r}\right|_{r=R}=0 .
\end{aligned}
$$

For solutions to problem (128), we have the inequality (see Lemma 1)

$$
\begin{array}{r}
\|u\|_{H_{\mu^{\prime}}^{2,1}\left(B_{R}^{T}\right)} \leq c\left(\left\|\frac{u_{, r}}{r}\right\|_{L_{2, \mu^{\prime}}\left(B_{R}^{T}\right)}+\left\|\frac{u}{r^{2}}\right\|_{L_{2, \mu^{\prime}}\left(B_{R}^{T}\right)}\right. \\
\left.+\|f\|_{L_{2, \mu^{\prime}}\left(B_{R}^{T}\right)}+\left\|u_{1}\right\|_{H_{\mu^{\prime}}^{1}\left(B_{R}\right)}\right) .
\end{array}
$$

The first two norms on the r.h.s. of (129) can be estimated in view of (124). Then we obtain some restriction on $\mu^{\prime}$. We have

$$
\left\|\frac{u_{, r}}{r}\right\|_{L_{2, \mu^{\prime}}\left(B_{R}^{T}\right)} \leq\left\|u_{, r}\right\|_{L_{2, \mu^{\prime}-1}\left(B_{R}^{T}\right)} \leq \alpha_{1}(T),
$$

where the last inequality holds for $\mu^{\prime}-1=\mu$, so $\mu^{\prime} \in(2-$ $(1 / 2) \sqrt{5}, 2+(1 / 2) \sqrt{5})$. Hence, $\mu^{\prime}$ can be chosen less than 1 . Similarly, the inequalities

$$
\left\|\frac{u}{r^{2}}\right\|_{L_{2, \mu^{\prime}}\left(B_{R}^{T}\right)} \leq\|u\|_{L_{2, \mu^{\prime}-2}\left(B_{R}^{T}\right)} \leq \alpha_{1}(T)
$$

hold for $\mu^{\prime}-2=\mu-1$ so $\mu^{\prime}-1=\mu$. Hence, the same restriction on $\mu^{\prime}$ as before is satisfied. Since the existence follows from the Galerkin method, Lemma 12 is proved.

In view of Lemma 12, we have the following.

Lemma 13. Assume that $w_{0} \in H_{\nu}^{2}\left(B_{R}\right), w_{1} \in H_{\nu}^{2}\left(B_{R}\right), v \in$ $(2,1+(1 / 2) \sqrt{5})$. Assume the Lipschitz continuity

$$
\begin{gathered}
\left|\psi_{, 9}(u)-\psi_{, 9}(v)\right| \leq c|u-v|, \\
\left|\psi_{, \eta}(u)-\psi_{, \eta}(v)\right| \leq c|u-v| .
\end{gathered}
$$

Then there exists a solution to problems (11)-(13) such that $w_{, t} \in$ $H_{v}^{2,1}\left(B_{R}^{T}\right)$ and

$$
\left\|w_{, t}\right\|_{H_{\nu}^{2,1}\left(B_{R}^{T}\right)} \leq c\left(\left\|w_{0}\right\|_{H_{\nu}^{2}\left(B_{R}\right)}+\left\|w_{1}\right\|_{H_{\nu}^{1}\left(B_{R}\right)}\right) .
$$

Proof. Applying Lemma 12 to problem (113) yields

$$
\begin{aligned}
\left\|w_{, t}^{(n+1)}\right\|_{H_{v}^{2,1}\left(B_{R}^{T}\right)} & \\
\leq c & \left\|w_{, r r}^{(n)}\right\|_{L_{2, \nu}\left(B_{R}^{T}\right)}+\left\|w_{, r}^{(n)}\right\|_{L_{2, \nu-1}\left(B_{R}^{T}\right)} \\
& \left.+\left\|w^{(n)}\right\|_{L_{2, \nu-2}\left(B_{R}^{T}\right)}+\left\|w_{1}\right\|_{H_{\nu}^{1}\left(B_{R}\right)}\right),
\end{aligned}
$$

where $v \in(2-(1 / 2) \sqrt{5}, 2+(1 / 2) \sqrt{5})$ and we used that

$$
\left|\psi_{, \vartheta \vartheta}\right| \leq c, \quad\left|\psi_{, \vartheta}\right| \leq c|\vartheta|, \quad\left|\psi_{, \eta}\right| \leq c|\eta| .
$$


In view of the relation

$$
w=\int_{0}^{t} w_{, t^{\prime}} d t^{\prime}+w_{0}
$$

we obtain from (134) the inequality

$$
\begin{aligned}
& \left\|w_{, t}^{(n+1)}\right\|_{H_{v}^{2,1}\left(B_{R}^{T}\right)} \\
& \quad \leq c T^{1 / 2}\left\|w_{, t}^{(n)}\right\|_{H_{v}^{2,1}\left(B_{R}^{T}\right)}+c\left\|w_{0}\right\|_{H_{\nu}^{2}\left(B_{R}\right)}+c\left\|w_{1}\right\|_{H_{\nu}^{1}\left(B_{R}\right)} .
\end{aligned}
$$

Hence, for sufficiently small $T$, we have

$$
\left\|w_{, t}^{(n)}\right\|_{H_{\nu}^{2,1}\left(B_{R}^{T}\right)} \leq c\left(\left\|w_{0}\right\|_{H_{\nu}^{2}\left(B_{R}\right)}+\left\|w_{1}\right\|_{H_{v}^{2}\left(B_{R}\right)}\right),
$$

for any $n \in \mathbb{N}$.

To show convergence of the sequence $\left\{w^{(n)}\right\}_{n \in \mathbb{N}}$ we introduce the differences

$$
W^{(n)}=w^{(n)}-w^{(n-1)},
$$

which are solutions to the problems

$$
\begin{gathered}
W_{, t t}^{(n+1)}-\frac{\mu_{0}}{r^{2}}\left(W_{, r t}^{(n+1)} r^{2}\right)_{, r}+\frac{\mu_{0}}{r^{2}} W_{, t}^{(n+1)} \\
=\frac{1}{r^{2}}\left[\left(\psi_{, 9^{(n)}}-\psi_{, 9^{(n-1)}}\right) r^{2}\right]_{, r}-\frac{1}{r}\left(\psi_{, \eta^{(n)}}-\psi_{, \eta^{(n-1)}}\right), \\
\left.W^{(n+1)}\right|_{t=0}=0,\left.\quad W_{, t}^{(n+1)}\right|_{t=0}=0,\left.\quad W_{, r}^{n+1}\right|_{r=R}=0 .
\end{gathered}
$$

To show convergence we multiply (140) by $W_{, t}^{(n+1)} r^{2 v}$ and integrate the result over $B_{R}$. Then we have

$$
\begin{aligned}
& \frac{1}{2} \frac{d}{d t} \int_{B_{R}}\left|W_{, t}^{(n+1)}\right|^{2} r^{2 v} d r+\mu_{0} \int_{B_{R}}\left|W_{, t r}^{(n+1)}\right|^{2} r^{2 v} d r \\
& \quad+\mu_{0} \nu(3-2 v) \int_{B_{R}}\left|W_{, t}^{(n+1)}\right|^{2} r^{2 v-2} d r \\
& =\int_{B_{R}} \frac{1}{r^{2}}\left[\left(\psi_{, 9^{(n)}}-\psi_{, 9^{(n-1)}}\right) r^{2}\right]_{, r} W_{, t}^{(n+1)} r^{2 v} d r \\
& \quad-\int_{B_{R}} \frac{1}{r}\left(\psi_{, \eta^{(n)}}-\psi_{, \eta^{(n-1)}}\right) W_{, t}^{(n+1)} r^{2 v} d r \equiv I_{1}+I_{2} .
\end{aligned}
$$

Integrating by parts in $I_{1}$ yields

$$
\begin{aligned}
I_{1}= & -\int_{B_{R}}\left(\psi_{, 9^{(n)}}-\psi_{, 9^{(n-1)}}\right) W_{, t r}^{(n+1)} r^{2 v} d r \\
& -2(\nu-1) \int_{B_{R}}\left(\psi_{, 9^{(n)}}-\psi_{, 9^{(n-1)}}\right) W_{, t}^{(n+1)} r^{2 v-1} d r \equiv I_{1}^{1}+I_{1}^{2},
\end{aligned}
$$

where

$$
\begin{aligned}
\left|I_{1}^{1}\right| \leq & \varepsilon_{1} \int_{B_{R}}\left|W_{, r t}^{(n+1)}\right|^{2} r^{2 v} d r \\
& +c\left(\frac{1}{\varepsilon_{1}}\right) \int_{B_{R}}\left|\psi_{, 9^{(n)}}-\psi_{, 9^{(n-1)}}\right| r^{2} d r,
\end{aligned}
$$

and the second integral in view of the assumption of the Lipschitz continuity is bounded by

$$
c \int_{B_{R}}\left|W_{, r}^{(n)}\right|^{2} r^{2 v} d r .
$$

Next, we examine

$$
\begin{aligned}
\left|I_{1}^{2}\right| & \leq c \int_{B_{R}}\left|\psi_{, 9^{(n)}}-\psi_{, 9^{(n-1)}}\right|\left|W_{, t}^{(n+1)}\right| r^{2 v-1} d r \\
& \leq \varepsilon_{2} \int_{B_{R}}\left|W_{, t}^{(n+1)}\right|^{2} r^{2 v-2} d r+c\left(\frac{1}{\varepsilon_{2}}\right) \int_{B_{R}}\left|W_{, r}^{(n)}\right|^{2} r^{2 v} d r .
\end{aligned}
$$

Finally, we estimate $I_{2}$,

$$
\begin{aligned}
\left|I_{2}\right| & \leq c \int_{B_{R}} \frac{1}{r^{2}}\left|W^{(n)}\right|\left|W_{, t}^{(n+1)}\right| r^{2 v} d r \\
& \leq \varepsilon_{3} \int_{B_{R}}\left|W_{, t}^{(n+1)}\right|^{2} r^{2 v-2} d r+c\left(\frac{1}{\varepsilon_{3}}\right) \int_{B_{R}}\left|W^{(n)}\right|^{2} r^{2 \nu-2} d r .
\end{aligned}
$$

Using the above estimates in (141) and assuming that $\varepsilon_{1}-\varepsilon_{3}$ are sufficiently small, we obtain

$$
\begin{gathered}
\frac{d}{d t} \int_{B_{R}}\left|W_{, t}^{(n+1)}\right|^{2} r^{2 v} d r+\mu_{0}^{\prime} \int_{B_{R}}\left|W_{, r t}^{(n+1)}\right|^{2} r^{2 v} d r \\
+\mu_{0} v(3-2 v) \int_{B_{R}}\left|W_{, t}^{(n+1)}\right|^{2} r^{2 v-2} d r \\
\leq c\left(\int_{B_{R}}\left|W_{, r}^{(n)}\right|^{2} r^{2 v} d r+\int_{B_{R}}\left|W^{(n)}\right|^{2} r^{2 v-2} d r\right),
\end{gathered}
$$

where $\mu_{0}^{\prime}<\mu_{0}$ and the same considerations as in (125)(127) are repeated. Integrating (147) with respect to time and employing the formula

$$
W^{(n)}(t)=\int_{0}^{t} W_{, t}^{(n)} d t
$$

we derive from (147) the inequality

$$
\begin{gathered}
\int_{B_{R}}\left|W_{, t}^{(n+1)}\right|^{2} r^{2 v} d r+\mu_{0}^{\prime} \int_{B_{R}^{T}}\left|W_{, r t}^{(n+1)}\right|^{2} r^{2 v} d r d t \\
+\mu_{0} \nu(3-2 v) \int_{B_{R}^{T}}\left|W_{, t}^{(n+1)}\right|^{2} r^{2 v-2} d r d t \\
\leq c T\left(\int_{B_{R}^{T}}\left|W_{, r t}^{(n)}\right|^{2} r^{2 v} d r d t+\int_{B_{R}^{T}}\left|W_{, t}^{(n)}\right|^{2} r^{2 v-2} d r d t\right) .
\end{gathered}
$$

Hence, we have that the sequence $\left\{w^{(n)}\right\}_{n \in \mathbb{N}}$ converges and then we show the existence of solutions to problems (11)-(13) on a small interval $(0, T)$. Since we have the estimate on the interval $(0, T)$ with arbitrary $T$, the above considerations can be repeated step by step.

In view of Lemmas 8 and 10 and Remark 9, the extension can be made under the restriction that $\nu \in(2,1+(1 / 2) \sqrt{5})$. This concludes the proof. 
Proof. Proof of the Main Theorem. In view of assumptions of Lemmas 8 and 10, Remark 9, and also by Lemmas 12 and 13, we have the first assertion of the theorem. In view of Lemma 3 and the Galerkin method, we prove the second assertion. In view of Lemmas 3 and 11, the third assertion follows. This concludes the proof.

\section{Acknowledgment}

W. M. Zajączkowski is partially supported by MNiSW Grant no. NN 201396937.

\section{References}

[1] G. Ponce, "Global existence of small solutions to a class of nonlinear evolution equations," Nonlinear Analysis: Theory, Methods \& Applications, vol. 9, no. 5, pp. 399-418, 1985.

[2] S. Kawashima and Y. Shibata, "Global existence and exponential stability of small solutions to nonlinear viscoelasticity," Communications in Mathematical Physics, vol. 148, no. 1, pp. 189-208, 1992.

[3] T. Kobayashi, H. Pecher, and Y. Shibata, "On a global in time existence theorem of smooth solutions to a nonlinear wave equation with viscosity," Mathematische Annalen, vol. 296, no. 2, pp. 215-234, 1993.

[4] I. Pawłow and W. M. Zajączkowski, "Global regular solutions to Cahn-Hilliard system coupled with viscoelasticity," Mathematical Methods in the Applied Sciences, vol. 32, no. 17, pp. 2197-2242, 2009.

[5] G. Andrews, "On the existence of solutions to the equation $u_{t t}=$ $u_{x x t}+\sigma\left(u_{x}\right)_{x}$, Journal of Differential Equations, vol. 35, no. 2, pp. 200-231, 1980.

[6] R. M. Christensen, Theory of Viscoelasticity, Academic Press, New York, NY, USA, 1971.

[7] A. C. Eringen, Continuum Physics, Academic Press, New York, NY, USA, 1978.

[8] W. Flügge, Viscoelasticity, Ginne (Blaisdell), Waltham, Mass, USA, 1967.

[9] W. Nowacki, Theory of Elasticity, Polish Scientific Publishers, Warsaw, Poland, 1970.

[10] W. Voigt, Theoretischen Physik, Veit, Leipzig, Germany, 1895.

[11] J. A. Gawinecki, "The Faedo-Galerkin method in thermal stresses theory," Commentationes Mathematicae, vol. 27, no. 1, pp. 83107, 1987.

[12] J. A. Gawinecki, "Global existence of solutions for non-small data to non-linear spherically symmetric thermoviscoelasticity," Mathematical Methods in the Applied Sciences, vol. 26, no. 11, pp. 907-936, 2003.

[13] J. A. Gawinecki and W. M. Zajączkowski, "Global non-small data existence of spherically symmetric solutions to nonlinear viscoelasticity in a ball," Journal of Analysis and Its Applications, vol. 30, no. 4, pp. 387-419, 2011.

[14] O. V. Besov, V. P. Il'in, and S. M. Nikolski, Integral Representation of Functions and Theorems of Imbedding, Nauka, Moscow, Russia, 1975 (Russian).

[15] O. A. Ladyzhenskaya, V. A. Solonnikov, and N. N. Uraltseva, Linear and Quasilinear Equations of Parabolic Type, Nauka, Moscow, Russia, 1967 (Russian).

[16] V. G. Maz’ya and B. A. Plamenevskiŭ, " $L_{p}$ estimates of solutions to elliptic boundary value problems in domains with edges,"
Trudy Moskovskogo Matematicheskogo Obshchestva, vol. 37, pp. 49-93, 1978 (Russian).

[17] V. A. Kondrat'ev, "Boundary value problems for elliptic equations in domains with conical or angular points," Trudy Moskovskogo Matematičeskogo Obščestva, vol. 16, pp. 209-292, 1967 (Russian).

[18] W. M. Zajączkowski, "Solvability of the heat equation in weighted Sobolev spaces," Applicationes Mathematicae, vol. 38, no. 2, pp. 147-171, 2011.

[19] W. M. Zajączkowski, "Existence of solutions to the (rot,div)system in $L_{p}$-weighted spaces," Applicationes Mathematicae, vol. 37, no. 2, pp. 127-142, 2010.

[20] W. M. Zajączkowski, "Solvability of the Poisson equation in weighted Sobolev spaces," Applicationes Mathematicae, vol. 37, no. 3, pp. 325-339, 2010. 


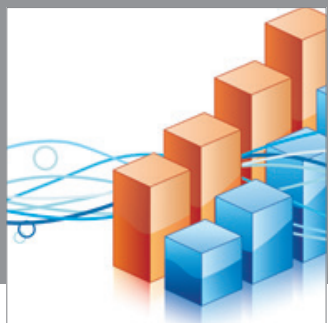

Advances in

Operations Research

mansans

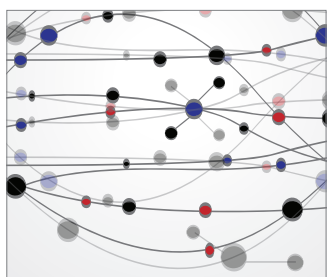

The Scientific World Journal
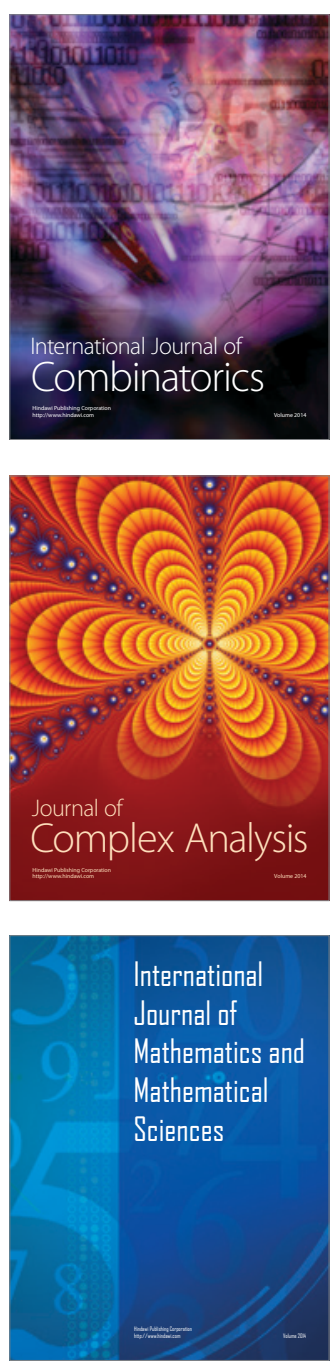
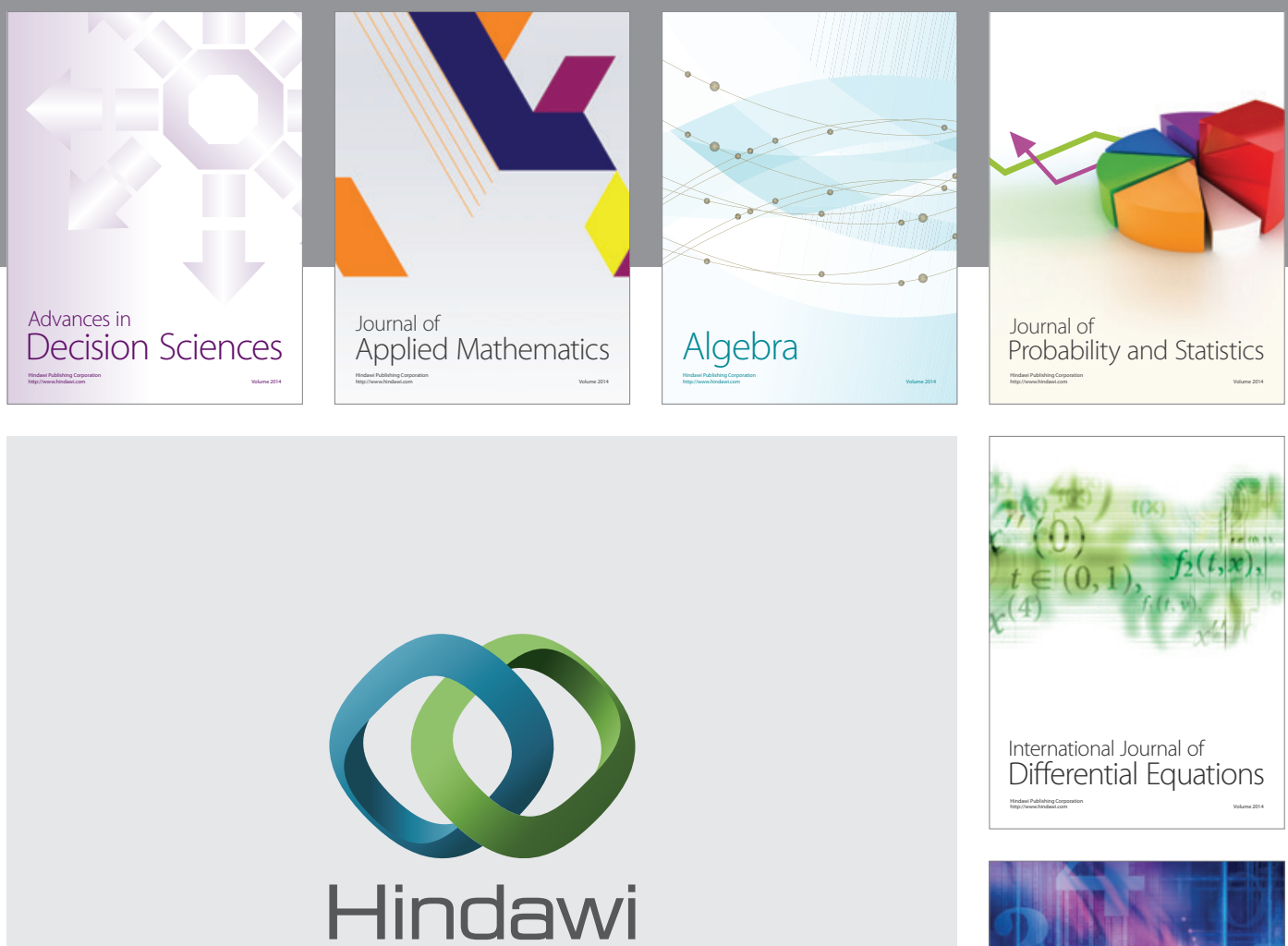

Submit your manuscripts at http://www.hindawi.com
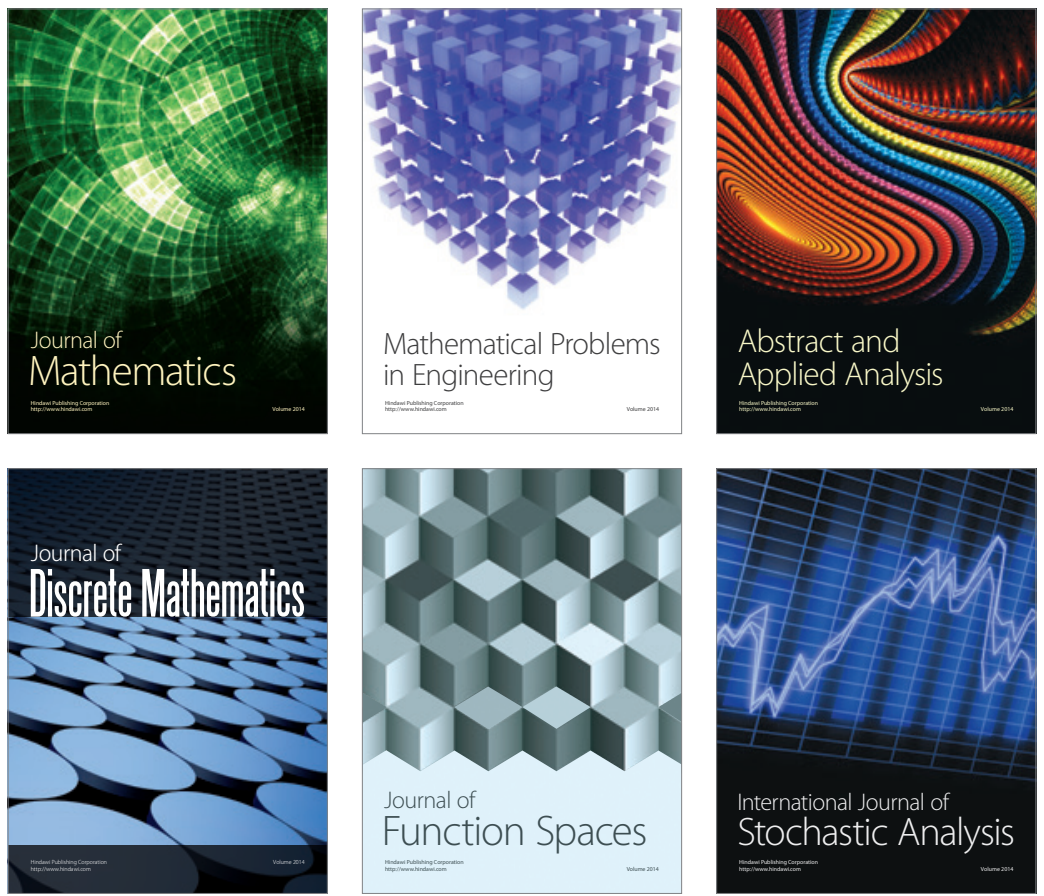

Journal of

Function Spaces

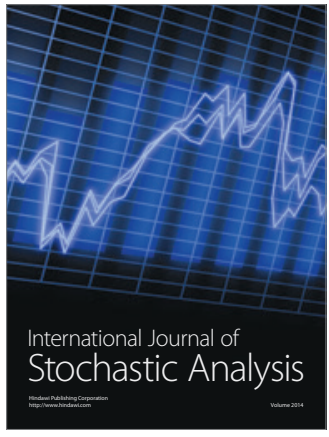

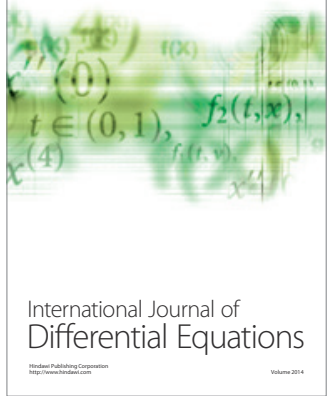
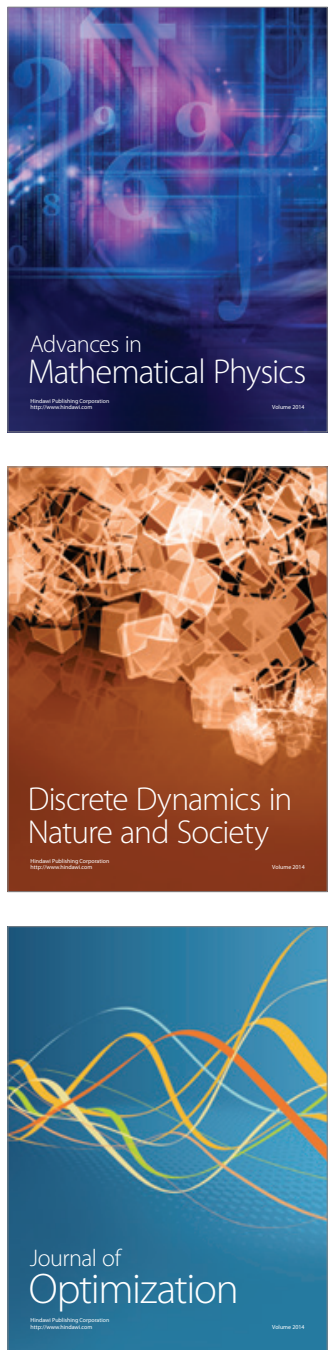